Journal for ImmunoTherapy of Cancer

\title{
Single-cell transcriptome analysis revealed a suppressive tumor immune microenvironment in EGFR mutant lung adenocarcinoma
}

\author{
Lei Yang (D) , ${ }^{1,2}$ Yun-Ting He, ${ }^{1}$ Song Dong, ${ }^{1}$ Xue-Wu Wei, ${ }^{1}$ Zhi-Hong Chen, ${ }^{1}$ \\ Bo Zhang, ${ }^{3}$ Wei-Dong Chen, ${ }^{3}$ Xiao-Rong Yang, ${ }^{1}$ Fen Wang (D) , Xue-Meng Shang, ${ }^{4}$ \\ Wen-Zhao Zhong, ${ }^{1}$ Yi-Long Wu (D) , ${ }^{1}$ Qing Zhou (D) ${ }^{1,2}$
}

To cite: Yang L, He Y-T, Dong S, et al. Single-cell transcriptome analysis revealed a suppressive tumor immune microenvironment in EGFR mutant lung adenocarcinoma. Journal for ImmunoTherapy of Cancer 2022;10:e003534. doi:10.1136/jitc-2021-003534

- Additional supplemental material is published online only. To view, please visit the journal online (http://dx.doi.org/10. 1136/jitc-2021-003534).

Accepted 29 November 2021

Check for updates

(C) Author(s) (or their employer(s)) 2022. Re-use permitted under CC BY-NC. No commercial re-use. See rights and permissions. Published by BMJ.

${ }^{1}$ Guangdong Lung Cancer Institute, Guangdong Provincial People's Hospital, Guangdong Academy of Medical Sciences, Guangzhou, China

${ }^{2}$ The Second School of Clinical Medicine, Southern Medical University, Guangzhou, China ${ }^{3}$ Novel Bioinformatics Co,

Shanghai, China

${ }^{4}$ Panovue Biological Technology Co, Beijing, China

Correspondence to

Professor Qing Zhou;

gzzhouqing@126.com

\section{ABSTRACT}

Backgrounds Immunotherapy is less effective in patients with epidermal growth factor receptor (EGFR) mutant non-small-cell lung cancer (NSCLC). Lower programmed cell death-ligand 1 (PD-L1) expression and tumor mutation burden (TMB) are reported to be the underlying mechanism. Being another important factor to affect the efficacy of immunotherapy, tumor microenvironment (TME) characteristics of this subgroup of NSCLC are not comprehensively understood up to date. Hence, we initiated this study to describe the specific TME of EGFR-mutant lung adenocarcinoma (LUAD) from cellular compositional and functional perspectives to better understand the immune landscape of this most common subtype of NSCLC.

Methods We used single-cell transcriptome sequencing and multiplex immunohistochemistry to investigate the immune microenvironment of EGFR-mutant and EGFR wild-type LUADs and determined the efficacy of immunotherapy. We analyzed single cells from nine treatment-naive samples and compared them to three post-immunotherapy samples previously reported from single cell perspective using bioinformatics methods.

Results We found that EGFR-mutant malignant epithelial cells had similar characteristics to the epithelial cells in non-responders. EGFR-mutant LUAD lacked $\mathrm{CD}^{+}$tissueresident memory (TRM) cells, which could promote tertiary lymphoid structure generation by secreting CXCL13. In addition, other cell types, including tumor-associated macrophages and cancer-associated fibroblasts, which are capable of recruiting, retaining, and expanding $\mathrm{CD} 8^{+}$ TRM cells in the TME, were also deficient in EGFR-mutant LUAD. Furthermore, EGFR-mutant LUAD had significantly less crosstalk between $T$ cells and other cell types via programmed cell death-1 (PD-1) and PD-L1 or other immune checkpoints compared with EGFR wild-type LUAD. Conclusions Our findings provide a comprehensive understanding of the immune landscape of EGFR-mutant LUAD at the single-cell level. Based on the results, many cellular components might have negative impact on the specific TME of EGFR-mutant LUAD through influencing $\mathrm{CD}^{+}$TRM. Lack of CD8 ${ }^{+}$TRM might be a key factor responsible for the suppressive TME of EGFR-mutant LUAD.

\section{INTRODUCTION}

Epidermal growth factor receptor (EGFR) mutation is the most common driver of non-small-cell lung cancer (NSCLC), which accounts for $40 \%-55 \%$ of lung adenocarcinomas (LUADs) in Asian patients. Tyrosine kinase inhibitors (TKIs) directed against EGFR variants are the standard treatment for EGFR-mutant NSCLC. However, the eventual acquisition of resistance is inevitable. There are few regimens capable of overcoming resistance to EGFR-TKIs.

Immune-checkpoint inhibitors (ICIs) have significantly improved the 5-year survival rate of patients with advanced lung cancer, ${ }^{1}$ and these agents are potentially curative even for late-stage NSCLC. Several ICIs have been approved as first-line or second-line treatments for patients with advanced NSCLC, but the indications do not encompass patients with sensitive EGFR mutations based on a series of large clinical trials. ${ }^{2}$ Patients with EGFR-mutant NSCLC have a lower response rate to immunotherapy compared with those with wild-type EGFR. Their progression-free survival and overall survival (OS) are not improved by ICIs even in patients with high programmed death-ligand 1 (PD-L1) expression. It is because PD-L1 expression cannot reflect the underlying $\mathrm{T}$ cell activity in EGFR driven NSCLC. ${ }^{3}$ Tumor mutation burden (TMB) level was reported to have a strong association with improved clinical outcome in patient with NSCLC. ${ }^{4}$ EGFR-mutant NSCLC was reported with comparatively lower TMB, which might be accountable for the poor efficacy of immunotherapy. ${ }^{5}$ However, being another essential impactor to immunotherapy, the tumor microenvironment (TME) in EGFR-mutation NSCLC is not comprehensively understood so far. 
In a subgroup analysis of patients with sensitive EGFR mutations in the IMpower150 study, improved OS with atezolizumab plus bevacizumab plus chemotherapy versus bevacizumab plus chemotherapy (BCP) was observed. ${ }^{6}$ However, no OS benefit was seen with atezolizumab plus chemotherapy versus BCP. The findings in the IMpower 150 study indicate that immunotherapy may benefit patients with EGFR-mutant NSCLC if the specific TME can be improved.

A recent study reported a patient with multiple nodules in the bilateral lung without metastases to other organ. ${ }^{7}$ Lung biopsy of the major nodule confirmed LUAD with pan-negative results of common driver gene mutations detected by next-generation sequencing (NGS) and higher PD-L1 expression tested by immunohistochemistry (IHC). This patient was EGFR-TKI naive and received three cycles of pembrolizumab as monotherapy followed by radical surgery. Radiological tumor assessment before surgery demonstrated significant shrinkage of nodule W2 $(-31.8 \%)$, whereas the other two nodules (W1 and W3) slightly increased in size $(+12.5 \%$ and $+8.3 \%$, respectively). This study investigated the response mechanism in multiple primary LUAD after neoadjuvant immunotherapy through multiomics analyses, including single-cell RNA sequencing (scRNA-seq). However, a lack of data on the baseline features of primary lung cancers before immunotherapy was an obvious limitation.

To explore the mechanism underlying the poor response to immunotherapy of patients with EGFRmutant LUAD, we performed scRNA-seq and multiplex IHC/immunofluorescence (mIHC/IF) to analyze the TME of treatment-naive patients with LUAD. We also analyzed the external scRNA-seq data ${ }^{7}$ of three primary LUAD nodules with different responses to pembrolizumab reported previously mentioned above, in terms of the TME before and after immunotherapy.

\section{METHODS}

Tumor tissues were freshly obtained from patients undergoing lung resection at the hospital. Nine samples were collected from eight patients (online supplemental table $1)$. The eligibility criteria were: confirmed LUAD, treatment naive, adequate surgical specimens of $>1 \mathrm{~cm}$ diameter, without other cancer. After resection, tumor-tissue samples were collected and immediately transferred for tissue preparation. Half of the tissues were subjected to single-cell isolation and the other half were subjected to nested PCR and MIHC for analysis of EGFR mutations and interested protein expression, respectively. The data of three samples from one patient with early stage multiple primary lung cancer, showing remarkable tumor shrinkage in a nodule and no response in two other nodules after treatment with three cycles of neoadjuvant pembrolizumab, were analyzed in this study. ${ }^{7}$ The dataset accession number is GSE146100 (Zhang et al).

The technological details of EGFR mutation analysis, single-cell dissociation, scRNA-seq and analysis, and
mIHC assays will be found in online supplemental materials. ScRNA-seq datasets generated in this study are available on the GEO database under the accession number GSE171145.

\section{RESULTS}

\section{Global immune landscape in LUAD}

To evaluate the immune landscape of the TME of LUAD based on EGFR mutation status, we collected nine tumor samples from eight treatment-naïve patients with LUAD for scRNA-seq and bioinformatic analyses (figure 1A). The nine samples comprised five EGFR-mutant and four EGFR wild-type LUADs (online supplemental table 1). Two EGFR-positive samples were from a patient with multiple nodules: one had EGFR L858R and the other had EGFR 19del.

We analyzed a total of 40,799 single cells, comprising 18,704 cells from four EGFR-negative samples and 22,095 cells from five EGFR-positive samples. Eight major cell types were identified in the dataset: epithelial cells, fibroblasts, endothelial cells, monocytic cells, T/natural killer (NK) cells, neutrophils, B/plasma cells, and mast cells. We conducted global cell-type annotations for each group and each patient (figure 1B, D and E, online supplemental figures 1 and 2). The proportions of these cell types in the TME differed significantly between the EGFR-positive and EGFR-negative groups (figure 1C,D).

$\mathrm{T} / \mathrm{NK}$ cells predominated and were present in similar proportions in both groups. However, the proportions of monocytic cells, mast cells, and endothelial cells were higher in the EGFR-positive group, and those of neutrophils and fibroblasts were higher in the EGFR-negative group (figure 1D).

\section{Epithelial cells of different EGFR status drive diversity in the TME}

A total of 9067 epithelial cells were obtained, which were further classified into 13 clusters (figure 2A). Given the association of cancer with large-scale chromosomal alterations, we used copy-number variation to classify epithelial cells as tumor or normal cells (figure 2D, online supplemental figure 3 ). We defined populations of $>50 \%$ tumor cells as tumor clusters and others as normal clusters. As a result, 10 clusters were annotated as tumor cells and 3 as normal cells (figure 2A). The total tumor cell proportions were similar in the EGFR-positive and EGFRnegative groups and most clusters comprised cells from both groups (figure 2B-D). However, according to the differential abundance sequencing (DASeq) analysis, clusters 1 and 5 were specific to the EGFR-positive group, and clusters $0,6,8$, and 11 to the EGFR-negative group.

Cluster 6, which was specific to the EGFR-negative group, showed upregulated expression of the interferon (IFN) alpha/gamma response and PI3K-AKT-MTORrelated signaling pathways (figure 2E). EGFR-positive tumor cells did not exhibit any particular characteristic in Gene Ontology (GO) analysis. However, the expression 

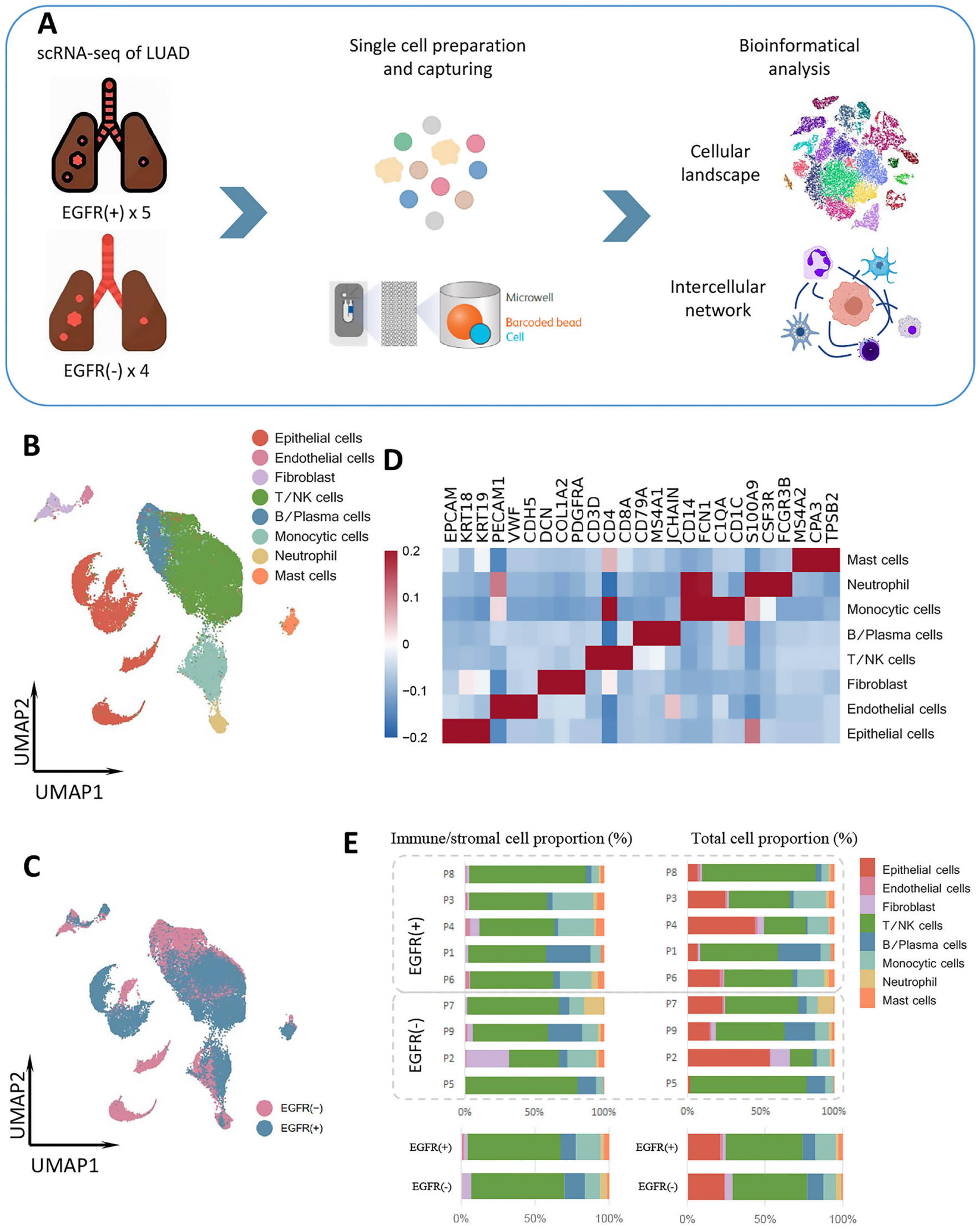

Figure 1 Global immune landscape and cell types in LUAD. (A) Workflow of the sample collection, preparation, sequencing, and bioinformatic analysis. (B) UMAP plot of the 40,799 total cells from treatment-naïve samples, color-coded by cell type. (C) UMAP plot of the 40,799 total cells from treatment-naïve samples, color-coded by group. (D) Heatmap of marker genes of all cell types. (E) Proportions of cell types in individual samples (above) and in different groups (bottom). EGFR, epidermal growth factor receptor; LUAD, lung adenocarcinoma; NK, natural killer; scRNA-seq, single-cell RNA sequencing. 

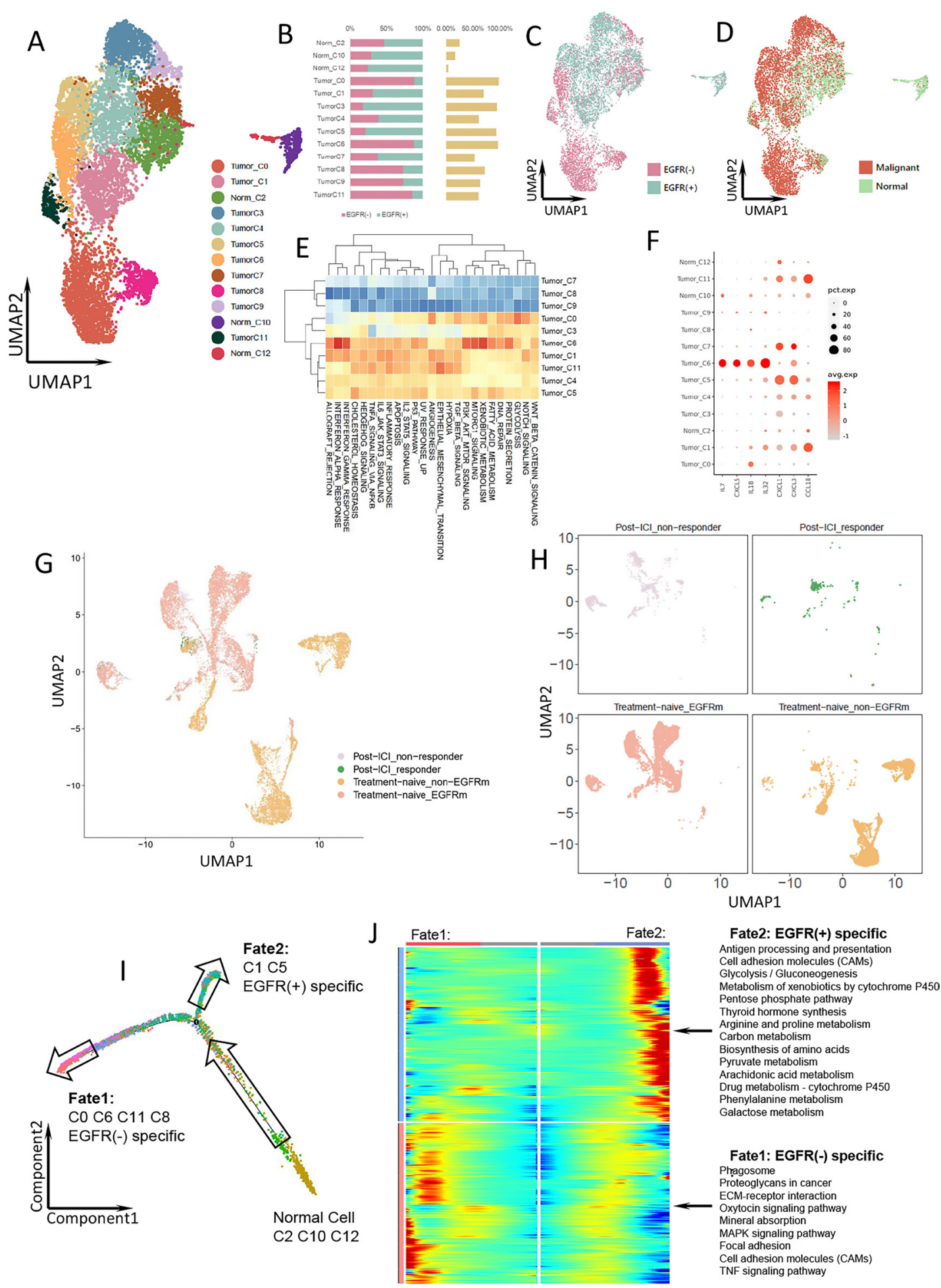

Figure 2 Epithelial cells with different EGFR mutation status in LUAD. (A) UMAP plot of 9067 epithelial cells from treatmentnaïve samples, divided into normal cell clusters and malignant cell clusters. (B) The proportion of each epithelial cell cluster in EGFR(-) (pink) and EGFR(+) (cyan) group. The yellow bar shows the proportion of each epithelial cell cluster in total epithelial cells. (C) UMAP plot of the epithelial cells from treatment-naïve samples, color-coded by group. (D) UMAP plot of the epithelial cells from treatment-naïve samples, color-coded by cell type. (E) Heatmap of the Qusage GO term enrichment/hallmark analysis of each cluster in epithelial cells. (F) Bubble plot of cytokine and IL expression among epithelial cell clusters. (G) UMAP plot of pooled epithelial cells in both treatment naïve and post-ICI treatment samples. (H) UMAP plot of epithelial cells from post-ICI responder, post-ICI non-responder, treatment-naïve EGFR-mutant and treatment-naïve EGFR(-) samples, respectively. (I) Pseudo-time plot of epithelial cells with different EGFR status in treatment-naïve samples exhibiting two differentiation trajectory fates. (J) Radiation plot showing significant differential expression for each fate. Genes with high expression are colored in red and genes with low expression are in blue. EGFR, epidermal growth factor receptor; GO, Gene Ontology; ICl, immune-checkpoint inhibitor; LUAD, IL, interleukin; LUAD, lung adenocarcinoma. 
of CCL18, CXCL1, and CXCL3 was higher in the EGFRpositive-specific clusters than in the EGFR-negative-specific clusters (figure 2F). CCL18 expression is associated with macrophage polarization and promotes regulatory $\mathrm{T}$ cell (Treg) recruitment, which leads to an immunosuppressive environment. ${ }^{8}$ CXCL3 induces myeloid-derived suppressor cell (MDSC) chemotaxis, ${ }^{9}$ and a high CXCL1 level is associated with enhanced recruitment of tumorassociated macrophages (TAMs) and cancer-associated fibroblasts (CAFs) in bladder cancer. ${ }^{10}$ Moreover, EGFRpositive-specific cluster 1 showed strong expression of interleukin (IL) $1 \beta$, an immune suppressive factor that promotes recruitment of MDSCs to tumor sites, ${ }^{11}$ and EGFR-negative-specific cluster 6 exhibited strong expression of CXCL5, IL-7, IL-18, and IL-32 (figure 2F). IL-7 is essential for $\mathrm{CD}^{+}$memory $\mathrm{T}$ cell proliferation ${ }^{12}$ and IL-18 is required for survival of activated $\mathrm{CD} 8^{+} \mathrm{T}$ cells and IFN- $\gamma$ production. ${ }^{13}$ Therefore, tumor cells with wild-type EGFR might have a greater ability to promote $\mathrm{CD} 8^{+} \mathrm{T}$ cell proliferation compared with tumor cells with mutant EGFR.

Comparing the epithelial cells from responsive and non-responsive samples after immunotherapy in public dataset to the treatment-naive samples with EGFR positive and negative status in our study, we found that epithelial cells from non-responsive nodules W1 and W3 after immunotherapy overlapped with EGFR-positive tumor cells in treatment-naïve samples, and cells from the responsive nodule W2 after immunotherapy overlapped with some normal and EGFR-negative tumor cells in treatment-naïve samples (figure $2 \mathrm{G}$ ). In addition, EGFRpositive-specific clusters 1 and 5 were identical to epithelial cells from non-responders (figure $2 \mathrm{H}$ ). These results are consistent with our hypothesis that EGFR-positive LUAD may poorly respond to immunotherapy.

The transcriptional differentiation trajectory of the epithelial cells revealed that the EGFR mutation drove the differentiation of normal epithelial cells to fate 1 (EGFR-negative group) or fate 2 (EGFR-positive group) (figure 2I). Analysis of the most highly expressed genes indicated that EGFR-mutant tumor cells had more such genes related to metabolic pathways (eg, glycolysis/gluconeogenesis, amino acid metabolism, and arachidonic acid metabolism) (figure 2J). Amino acid catabolism is crucial for suppressing the functions of T cells and other immune cells. ${ }^{14}$ Also, glucose deprivation inhibits the production of IFN- $\gamma$ and the effector functions of $\mathrm{CD} 8^{+}$ T cells. ${ }^{15}$ The metabolic features may also have negative impact on the TME in EGFR-mutant LUAD.

\section{CD8 $^{+}$TRM cells are enriched and activated in EGFR wild-type LUAD and $\mathrm{CD}^{+}{ }^{+}$TRM cells might promote tertiary lymphoid structure generation through CXCL13 secretion}

$\mathrm{T}$ and NK cells were classified into $\mathrm{CD} 8^{+} \mathrm{T}$ cells, $\mathrm{CD} 4^{+}$ $\mathrm{T}$ cells, and NK cells (online supplemental figure 4A,B). Next, $\mathrm{CD} 8^{+}$and $\mathrm{CD} 4^{+} \mathrm{T}$ cells were reclustered into nine and eight subgroups, respectively (figure 3A,C). The proportions of $\mathrm{CD}^{+}$TRM cells, $\mathrm{CD}^{+}$cycling $\mathrm{T}$ cells which highly expressed MKI67, CD4 $4^{+}$TRM cells, and Th1-like cells were higher in the EGFR-negative group (figure 3B,D). The distributions of these clusters overlapped with the area enriched in EGFR-negative cells (online supplemental figure 4C,D). We analyzed the marker genes of active $\mathrm{CD} 8^{+} \mathrm{T}$ cells and found that their expression was significantly higher in the EGFR-negative group compared with EGFR-positive group (online supplemental figure $4 \mathrm{E}$ ). This result was supported by previous report that EGFR mutants had a high frequency of inactive tumor infiltrating lymphocytes (TILs), which was concluded from IF staining test of CD3, Ki67, and granzyme B co-expression. ${ }^{3}$

CD103 is a marker expressed on the surface of TRM cells, and $\mathrm{CD} 103^{+} \mathrm{CD}^{+}$TRM cells are associated with improved survival after immunotherapy in many types of cancer. ${ }^{16} 17$ Furthermore, many suppressive immune checkpoints, such as programmed cell death protein 1 (PD-1), lymphocyte activating 3 (LAG3), and T cell immunoglobulin and mucin domain-containing protein 3 (TIM3) are highly expressed by TRM cells. ${ }^{16} 18$ Our mIHC results showed a higher proportion of $\mathrm{CD} 103^{+} \mathrm{CD}^{+} \mathrm{T}$ cells in the EGFRnegative group (figure 3E). In addition, the expression of PD-1, LAG3, and TIM3 was higher in $\mathrm{CD}^{+}{ }^{+} \mathrm{T}$ cells in the EGFR-negative group, especially in the cancer nest area (online supplemental figure $4 \mathrm{~F}$ ). In scRNA-seq, both inhibitory (PDCD1, CTLA4, LAG3, TIGIT, HAVCR2, and CD96) and activation (TNFRSF9, ICOS, and CD226) immune checkpoints were more highly expressed by CD8 ${ }^{+}$TRM cells, which were absent in the EGFR-positive group (figure $3 \mathrm{~F}$ ). These results showed that $\mathrm{CD} 8^{+} \mathrm{TRM}$ cells were enriched in the TME of EGFR wild-type LUAD, consistent with the mIHC data.

On investigating the cytokines secreted by $\mathrm{T}$ cells, we found that CXCL13 expression was higher in CD8 ${ }^{+}$TRM, $\mathrm{CD}^{+}$cycling $\mathrm{T}$ cells and $\mathrm{CD} 4^{+}$Th1-like cells in the EGFRnegative group (figure $3 \mathrm{G}$, online supplemental figure $4 G)$. CXCL13 mediates recruitment of B cells to tumors and is essential for the formation of tertiary lymphoid structure (TLS), the density of which is a positive predictive factor for a response to immunotherapy in several cancer types. ${ }^{19} 20$ The mIHC results revealed that CXCL13 expression in $\mathrm{CD}^{+} \mathrm{T}$ cells, and the density of TLSs, were significantly higher in the EGFR-negative than EGFRpositive group (figure $3 \mathrm{E}, \mathrm{H}$ ); the difference was more significant in the stromal region, suggesting that CXCL13 may promote TLS formation in the tumor stromal region. Nevertheless, CXCL13 expression in total cells was higher in the EGFR-positive group than EGFR-negative group. The contrary results of CXCL13 expression by $\mathrm{CD} 8^{+}$ $\mathrm{T}$ cells and total cells indicated the necessity to analyze CXCL13 in specific cell population rather than in total cells when exploring the characteristics of TME. The higher proportion of TRM cells in the EGFR-negative group might mediate the formation of TLS by secreting CXCL13. By contrast, T cells in the EGFR-positive group expressed more immune-suppressive cytokines such as CCL4, CXCL2, CXCL3, CXCL17, and IL-1 $\beta$, than those 
A
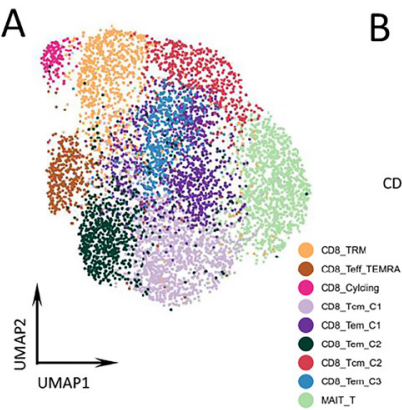

B

E
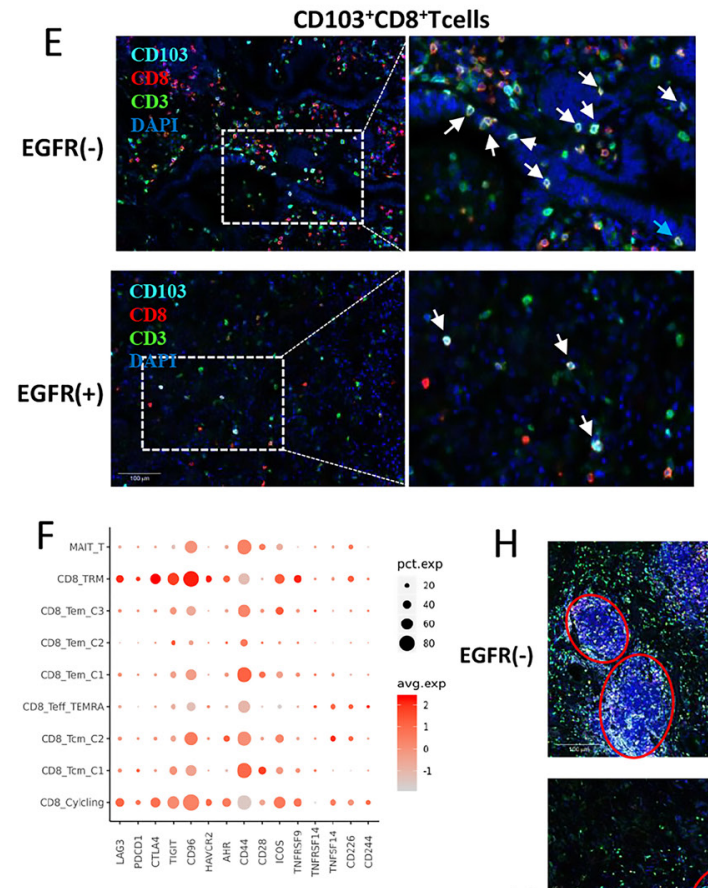

G

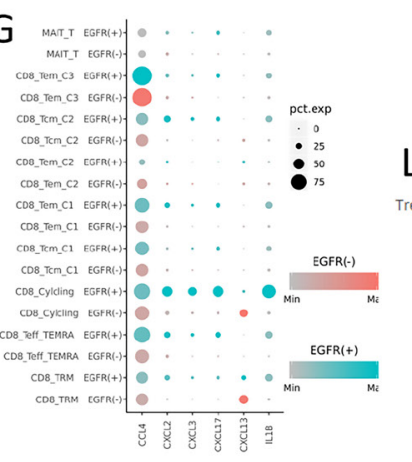

$\mathrm{H}$
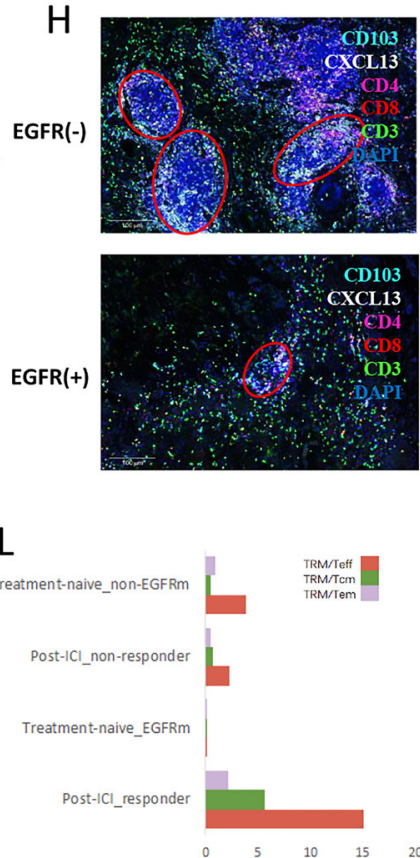

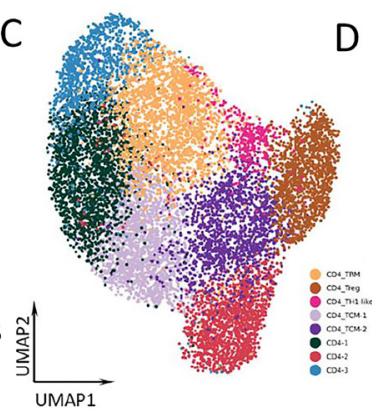

D
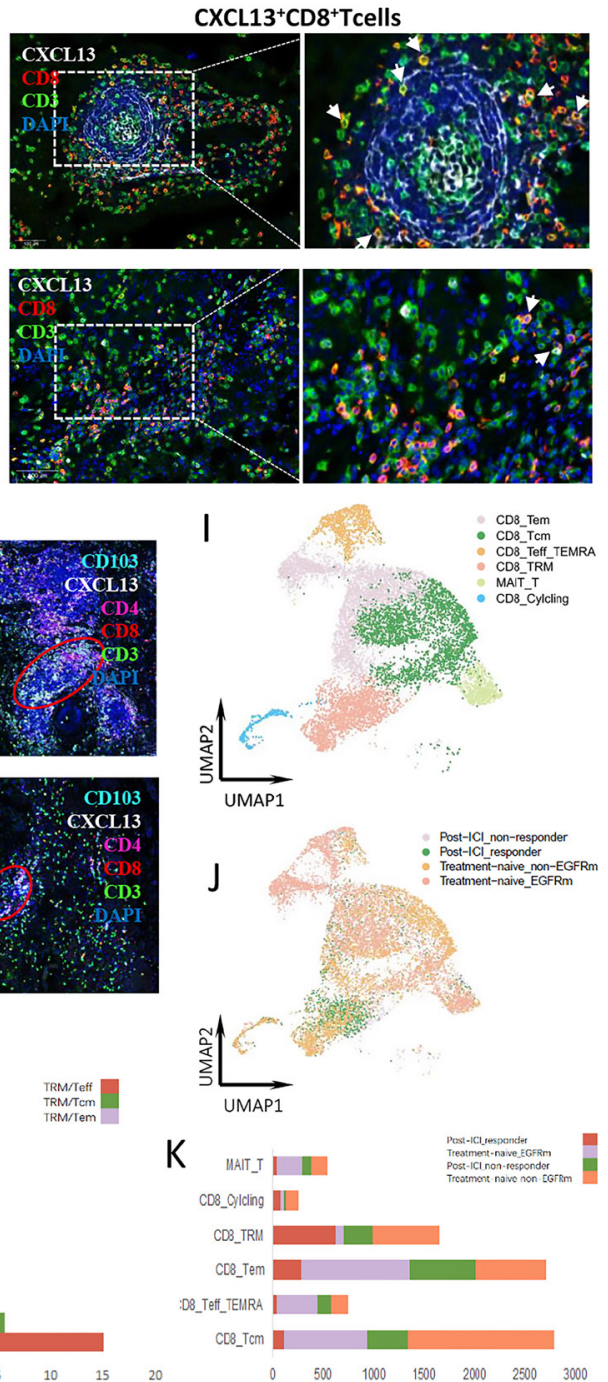

Figure $3 \mathrm{~T}$ and NK cell clusters and functions in LUAD. (A) UMAP plot of $6568 \mathrm{CD} 8^{+} \mathrm{T}$ cells from treatment-naïve samples. (B) The proportion of each CD8 ${ }^{+}$Tcell cluster in EGFR(-) (pink) and EGFR(+) (cyan) group. (C) UMAP plot of $11,755 \mathrm{CD}^{+} \mathrm{T}$ cells from treatment-naïve samples. (D) The proportion of each CD4 ${ }^{+}$Tcell cluster in EGFR(-) (pink) and EGFR(+) (cyan) group. (E) Expression of CD103 (left) and CXCL13 (right) by CD8 ${ }^{+}$T cells in the EGFR(-) and EGFR(+) TME. The TLS structure with $\mathrm{CXCL} 13^{+} \mathrm{CD}^{+} \mathrm{T}$ cells was also displayed in EGFR(-) TME. The TLS structure was surrounded by CXCL13 ${ }^{+} \mathrm{CD} 8^{+} \mathrm{T}$ cells. (F) Bubble plot of immune checkpoint receptor expression among CD8 ${ }^{+}$Tcell clusters. (G) Bubble plot of cytokine and IL expression among CD8 ${ }^{+}$Tcell clusters from different group. (H) The density of TLS in the EGFR(-) and EGFR(+) TME. (I) UMAP plot of pooled $\mathrm{CD}^{+} \mathrm{T}$ cells in both treatment-naïve and post-treatment samples. All pooled $\mathrm{CD} 8^{+} \mathrm{T}$ cells were annotated and divided into 6 main cell types. (J) UMAP plot of pooled $C D 8^{+} \mathrm{T}$ cells from post-ICl responder, post-ICI non-responder, treatment-naïve EGFR-mutant, and treatment-naïve EGFR-negative samples, respectively. (K) The proportion of different $\mathrm{CD}^{+} \mathrm{T}$ cell cluster from post-ICI responder, post-ICI non-responder, treatment-naïve EGFR-mutant, and treatment-naïve EGFR(-) samples, respectively. (L) The ratio of CD8 ${ }^{+}$TRM cells to other T cells in post-ICl responder, post-ICI non-responder, treatment-naïve EGFR-mutant, and treatment-naïve EGFR-negative samples, respectively. EGFR, epidermal growth factor receptor; ICI, immune-checkpoint inhibitor; IL, interleukin; NK, natural killer; TLS, tertiary lymphoid structure; TME, tumor microenvironment. 
in the EGFR-negative group (figure 3G). These cytokines function to recruit immune-suppressive cells, such as MDSCs. ${ }^{9} 11$ 21-23

To validate our findings, external scRNA-seq data from three samples subjected to pembrolizumab treatment were analyzed. In the combined cell pool, both the EGFRpositive and EGFR-negative groups had many $\mathrm{CD} 8^{+} \mathrm{T}$ cells; similar proportions of subclusters were seen between the two groups, including CD8 ${ }^{+}$effector T cells (Teffs), which are considered effective tumor-killing T cells. Different from the other subclusters, CD8 ${ }^{+}$TRM cells were seen in higher proportions in the EGFR-negative group than EGFR-positive group (figure 3I-K). CD8 ${ }^{+}$TRM cells were found in each sample from the EGFR-negative group. Moreover, $\mathrm{CD}^{+} \mathrm{TRM}$ cells were the major $\mathrm{CD}^{+} \mathrm{T}$ cells of the responsive nodule (figure $3 \mathrm{I}-\mathrm{J}$ ). We found that the TRM/Teff ratio was higher in the EGFR-negative group than EGFR-positive group, for the treatment-naive samples. After immunotherapy, TRM/Teff ratio was increased in both responsive and non-responsive samples, and the increase in responsive samples was greater than in the non-responsive samples (figure $3 \mathrm{~L}$ ). $\mathrm{CD}^{+} \mathrm{TRM}$ cells significantly expanded during anti-PD-1 treatment of patients with melanoma and lung cancer, ${ }^{16}{ }^{17}$ consistent with our analyses. The significantly increased TRM/ Teff ratio in responsive tumors after immunotherapy indicated that $\mathrm{CD}^{+}$TRM expansion and activation might be associated with the response of immunotherapy. $\mathrm{CD}^{+}$ TRM enrichment in baseline tumor tissue might be one of the predicting factors of the response to ICIs.

Our scRNA and mIHC results revealed the absence of $\mathrm{CD}^{+}$TRM cells, lower expression of CXCL13, and higher expression of immune suppressive factors by $\mathrm{T}$ cells in EGFR-positive LUAD. These characteristics of T cell negatively modified the TME of EGFR-mutant LUAD.

\section{Macrophages might recruit and expand $\mathrm{CD}^{+} \mathrm{TRM}^{\mathrm{T}}$ cells in the TME directly and indirectly in EGFR-negative LUAD}

Monocytes were classified and annotated as dendritic cells (DCs), macrophages, and other monocytes (online supplemental figure 5A,B). Macrophages were classified as TAMs and alveolar macrophages, and divided into eight subclusters (figure 4A,D). The proportions of alveolar macrophages C1 and C3 were higher in the EGFRpositive group, and that of the CHIT1_TAM cluster was higher in the EGFR-negative group (figure 4B). DASeq analysis demonstrated that EGFR-positive specific areas overlapped with alveolar macrophages C1 and C3, and EGFR-negative specific areas overlapped with CHIT1_ TAM (figure 4C).

Generally, CCL2, CCL13, GDF15, CCL23, and CXCL17 were highly expressed by macrophages in the EGFRpositive group, and CXCL9, CXCL10, CCL3, CXCL5, and CXCL12 were highly expressed by those in the EGFRnegative group (figure 4E). CCL2 mediates recruitment of MDSCs to tumor sites, ${ }^{24}$ and CCL13 is a marker of M2-like macrophages. ${ }^{25}$ GDF15 reportedly suppresses macrophage surveillance, inhibits cytotoxic T-lymphocyte activation by DCs, and regulates cellular metabolism. ${ }^{26}$ CCL23 upregulates KDR/flk-1 receptor expression and potentiates vascular endothelial growth factor (VEGF) action in angiogenesis. ${ }^{27}$ CXCL17 is a myeloid cellattracting chemokine and can increase the number of alveolar macrophages. ${ }^{22}$ These cytokines may shape an immune-suppressive microenvironment in EGFR-mutant LUAD. By contrast, CXCL9 and CXCL10 are IFN- $\gamma$-inducible chemokines. In the TME, secretion of CXCL9 and CXCL10 attracts activated effector T lymphocytes with antitumor reactivity via CXCL9-CXCR3 binding. ${ }^{28}{ }^{29}$ TAMs may recruit TRM cells via CXCL9 expression and are associated with strong TRM tumor infiltration and better outcomes. ${ }^{30}$ Macrophage-derived CXCL9 and CXCL10 are required for antitumor immune responses following immune checkpoint blockade. ${ }^{31}$ CCL3 has the ability to recruit cytotoxic TIL and interact with B cells synergistically. ${ }^{32}$

When evaluating previous data after immunotherapy, the integrated macrophages were mainly divided into alveolar macrophages and TAMs (figure 4F). The alveolar macrophage distribution was mixed, including cells from both EGFR-positive and EGFR-negative groups. However, the TAM distribution was clearly distinguished by the EGFR genetic status in treatment-naïve samples and the response to immunotherapy in post-treatment samples. Then, we analyzed TAMs from the posttreatment samples. TAMs from responsive samples highly and specifically expressed CCL7, or CXCL9 and CXCL10, and overlapped with TAMs from the EGFR-negative treatment-naïve group (figure 4G, online supplemental figure 5C). These TAMs were further annotated as CCL7_ TAMs and CXCL9_TAMs (figure 4H). CCL7 can promote the subsequent expansion of $\mathrm{CD}^{+}$and $\mathrm{CD}^{+} \mathrm{T}$ cells in NSCLC mouse models and were positively correlated with CD103 in human NSCLC tumor biopsies, ${ }^{33}$ which indicated that macrophages with higher CCL7 expression in the EGFR-negative group might facilitate TRM expansion. Comparing the clusters from combination pool to those from treatment-naive samples, CCL7_TAMs and CXCL9_TAMs were mostly derived from CHIT1_ TAMs and CCL13_TAMs in the EGFR-negative group (figure 4I).

We further analyzed the crosstalk between TAMs and other cells in treatment-naive samples. CHIT1_TAM can interact with all types of T cells, including TRM cells, by secreting CCL7 to bind the related receptors expressed by $\mathrm{T}$ cells (figure $4 \mathrm{~J}$ ). The same crosstalk manner was also seen in antigen presenting CAF (apCAF). The cell-cell interaction network analysis showed that macrophages were in the center of the network of all cell types in the TME. The CHIT1_TAM in the EGFR-negative group showed more frequent communication with other immune cells (figure 4K). These results indicated that CHIT1_TAM might have strong modulatory abilities in the TME of EGFR-negative LUAD and could be advantageous for mediating TRM activation and retention, both directly and indirectly. However, this population 

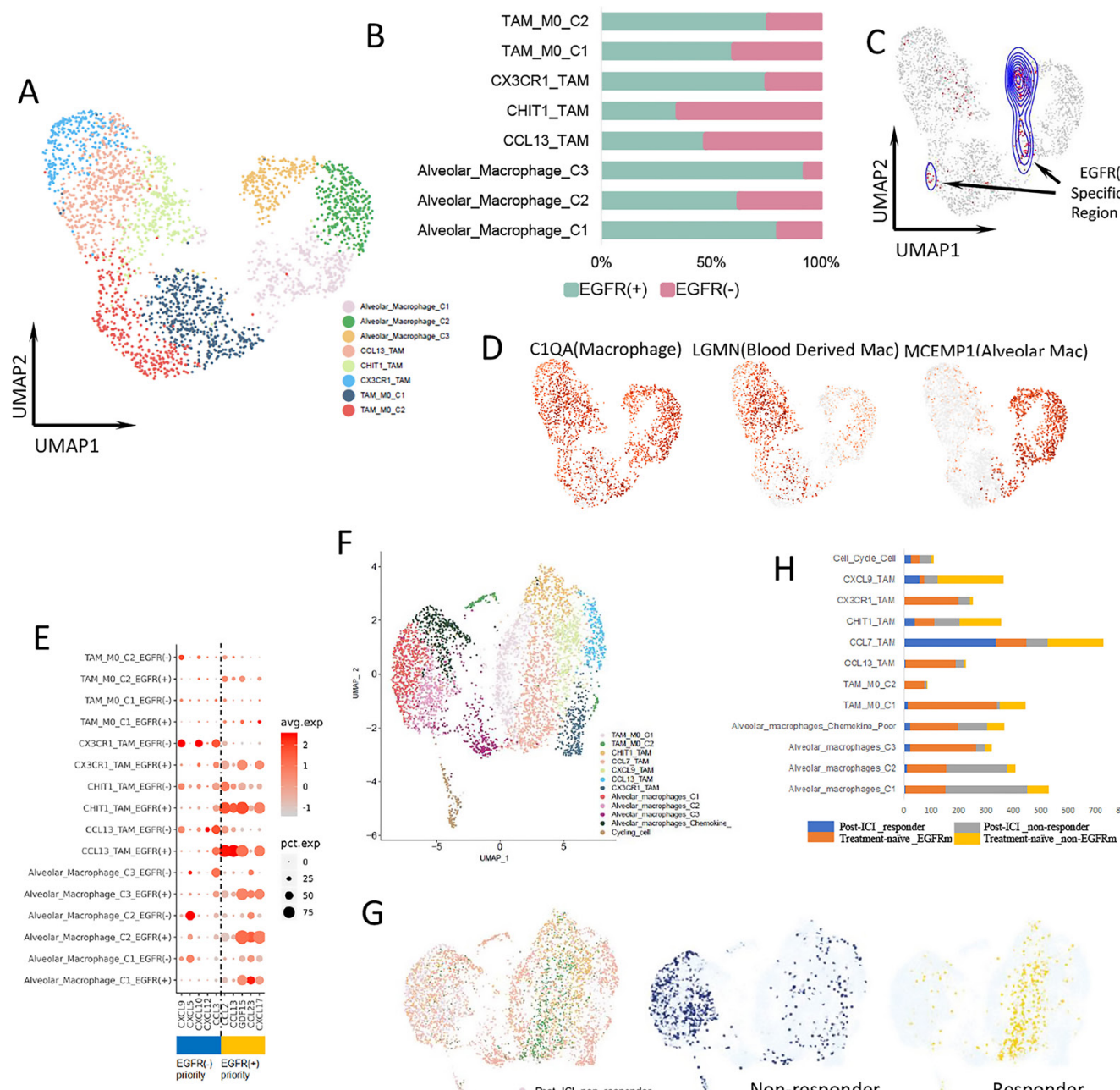

F
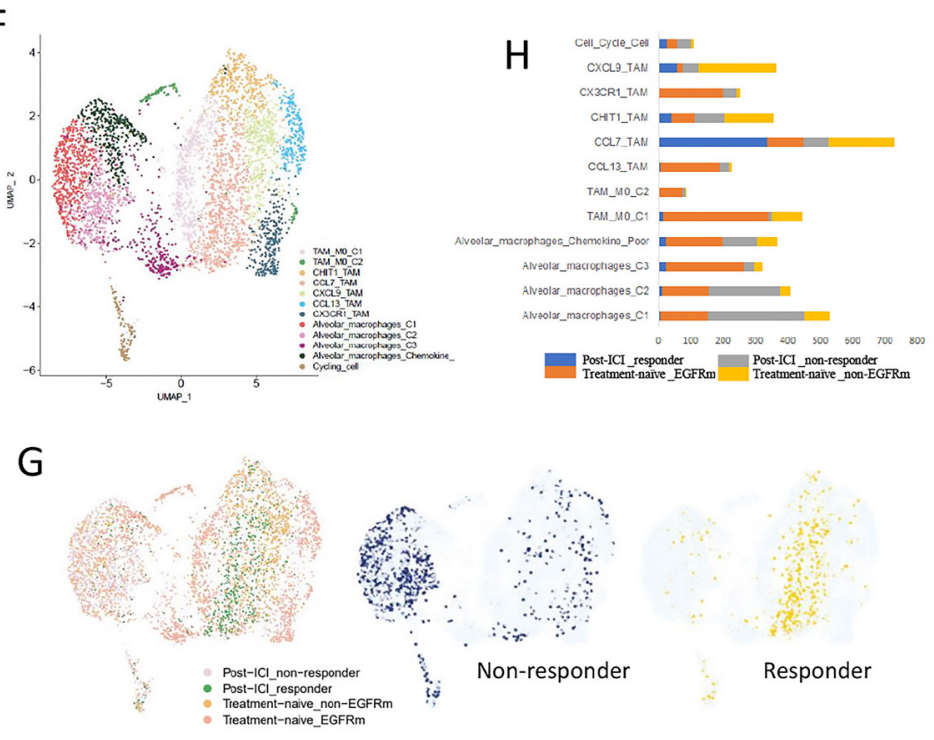

Responder
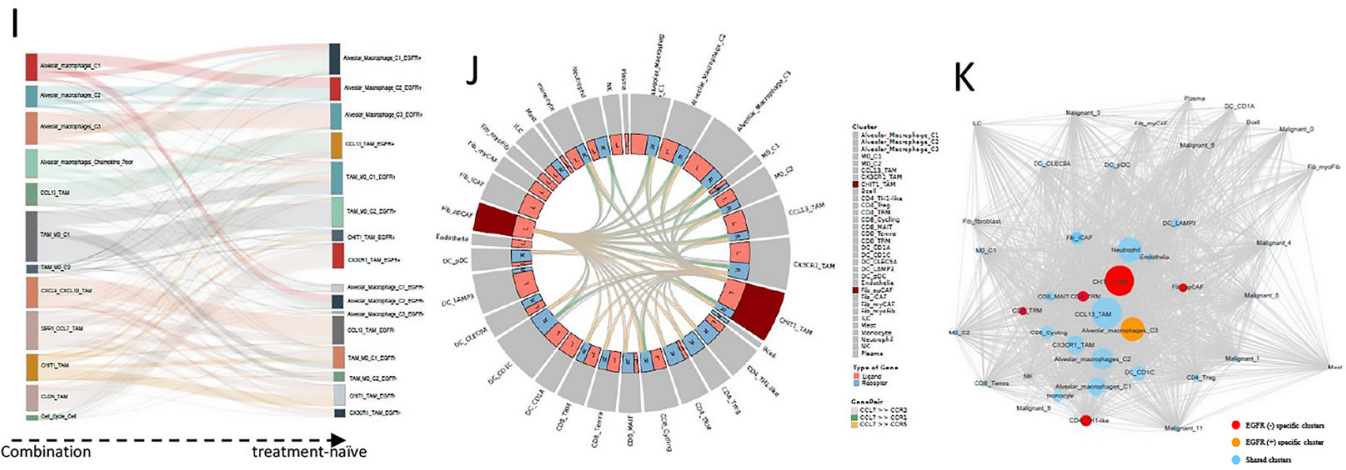

Figure 4 Macrophage expression differs by EGFR mutation status. (A) UMAP plot of 2649 macrophages from treatmentnaïve samples. (B) The proportion of each macrophage cluster in EGFR(-) (pink) and EGFR(+) (cyan) group. (C) UMAP plot of macrophages from EGFR(+)-specific region calculated by DASeq. (D) Expression of marker genes for macrophages, bloodderived macrophages, and alveolar macrophages. (E) Bubble plot of cytokine expression among macrophage clusters from different group. (F) UMAP plot of pooled macrophages in both treatment-naïve and post-treatment samples. (G) UMAP plot of pooled macrophages from post-ICI responder, post-ICI non-responder, treatment-naïve EGFR-mutant, and treatmentnaïve EGFR-negative samples, respectively (left). The macrophages from responder (middle) and non-responder (right) were separately distributed. $(\mathrm{H})$ The proportion of different macrophage cluster from post-ICI responder, post-ICI non-responder, treatment-naïve EGFR-mutant, and treatment-naïve EGFR-negative samples, respectively. (I) Macrophage clusters comparation between combination samples and treatment-naïve samples. (J) CHIT1_TAM interacted with other immune cells, including CD8 ${ }^{+} \mathrm{T}$ cells through CCL7 and its receptors binding. (K) Cellular interaction network for all LUAD cell types constructed based on the CellPhoneDB results. Lines represent the relationship between two clusters in terms of cytokine expression. Node size reflects the strength of the relationship. Red nodes represent EGFR(-)-specific clusters, orange nodes represent EGFR(+)specific clusters, and blue nodes represent sharing clusters from both groups. EGFR, epidermal growth factor receptor; ICl, immune-checkpoint inhibitor; TAM, tumor-associated macrophage. 
of macrophages is lacking in the microenvironment of EGFR-mutant LUAD.

\section{CAFs might help convert CD8 ${ }^{+} \mathrm{T}$ cells into $\mathrm{CD8}{ }^{+}$TRM cells in the TME of EGFR wild-type LUAD}

Fibroblasts were classified into eight clusters and annotated as fibroblasts, myofibroblasts, and CAFs (figure 5A,B). CAFs are a central element of the TME and interact with cancer cells and other components of the TME. We divided CAFs into myofibroblastic CAFs (myCAFs), apCAFs, and inflammatory CAFs (iCAFs) (figure 5C,D). The proportions of apCAFs, myCAFs-1, and myCAFs-2 were higher in the EGFR-negative group than EGFR-positive group. The proportion of iCAFs was similar in both groups (figure 5E). DASeq also showed that the distribution of apCAFs, myCAFs-1, and myCAFs-2 overlapped with that of EGFR-negative-specific cells (figure 5F). Hallmark analysis of apCAFs revealed that this cluster was enriched in proinflammatory pathways (figure 5G).

Further comparison of treatment-naïve and posttreatment samples indicated that fibroblasts were present in non-responsive samples, but rarely in responsive samples, after ICI treatment. In post-treatment samples, apCAFs were absent in both responders and non-responders. The major and minor subtypes in nonresponsive samples were $\mathrm{LEPR}^{+} \mathrm{CAFs}$ and myCAFs, respectively (figure $5 \mathrm{H}, \mathrm{I}$, online supplemental figure 6B). Thus, residual $\mathrm{LEPR}^{+} \mathrm{CAFs}$ might contribute to immune resistance. LEPR is a marker of mesenchymal stem cells (MSCs), ${ }^{34}$ and $\mathrm{LEPR}^{+}$CAFs can be considered MSC-like CAFs. iCAFs and partial fibroblasts in treatment naïve samples were correlated with $\mathrm{LEPR}^{+} \mathrm{CAF}$ in combination pool (figure 5J). Further analyses showed that MMP2, CXCL14, CXCL12, FAP, TNFSF13B, BMP5, and HGF were specifically expressed by these residual CAFs (figure 5K). MMP2 may contribute to tumor immune escape, as it is positively correlated with Tregs and most inhibitory immune checkpoints across multiple cancer types. Inhibiting MMP2 could enhance the therapeutic efficacy of PD-1 blockade. ${ }^{35}$ Fibroblast-derived CXCL14 increases the expression of mesenchymal markers and induces the epithelial-mesenchymal transition and lung metastasis. ${ }^{36}$ CAFs can attract Tregs and enhance their capacity to inhibit Teff proliferation by secreting CXCL12. ${ }^{37} \mathrm{FAP}^{+}$CAF is the principal source of CXCL12 in the tumor. Targeting $\mathrm{FAP}^{+}$CAF-derived CXCL12 could improve immunotherapy. ${ }^{38}$ TNFSF13B promotes the expansion of Tregs in the TME. ${ }^{39}$ BMP5 is a key regulator of basal stem/progenitor cell maintenance. ${ }^{40}$ HGF plays an immunosuppressive role and constitutes a mechanism of primary and acquired resistance to cancer immunotherapy. ${ }^{41}$

In treatment-naïve samples, iCAFs highly expressed LEPR and CXCL12, and some markers of MSCs ${ }^{42}$ were also highly expressed by myCAFs, including ITGB1, CD44, ISLR, and THY1 (online supplemental figure 6C). These MSC-like CAFs might interfere with the immune response. However, TGF- $\beta$ signaling is essential for the differentiation of MSCs into CAFs in the TME ${ }^{43}$ We found that TGF- $\beta$ was expressed more by iCAFs and myCAFs from the EGFR-negative group than EGFR-positive group (online supplemental figure 6C). The abundant TGF- $\beta$ within the TME could also induce the expression of CD103 on the CD8 ${ }^{+} \mathrm{T}$ cell surface. ${ }^{44}$ Thus, CAFs from the EGFR-negative group might have the ability to induce $\mathrm{CD}^{+} \mathrm{T}$ cells to convert into $\mathrm{CD} 8^{+} \mathrm{TRM}$ cells, which could support our other findings in $\mathrm{T}$ cells.

\section{B cells construct the TLS in the TME of EGFR-negative LUAD}

$\mathrm{B}$ cells were divided into 10 clusters and annotated as plasma and B cells (figure 6A,B). The proportion of plasma cells was higher in the EGFR-negative group than in the EGFR-positive group (figure 6C). Plasma-cell expression of the immune suppressive factors CXCL17 and GDF15 was higher in the EGFR-positive group, ${ }^{22} 26$ while that of CCL18 and GRN, which is linked to the activation of $\mathrm{CAF}^{45}$ were higher in the EGFR-negative group (figure 6D).

$\mathrm{B}$ cells are the most essential and fundamental components of TLSs. Cell-cell interaction analysis showed that B cells could be recruited by CHIT1_TAM, iCAF, and $\mathrm{LAMP}^{+} \mathrm{DC}$, through various cytokine ligands binding with receptors (figure 6E). B cells expressing CCR7 could be recruited by CHIT1_TAM secreting CCL21. CCR7 was more highly expressed by B cells from the EGFRnegative group, while CCL21 was specifically expressed by CHIT1_TAM from the EGFR-negative group (figure 6F). Furthermore, according to a previous report, CD79A plays an important role in germinal center initiation and TLS formation, and was considered the signature gene of TLS. ${ }^{46}$ In our samples, CD79A was more highly expressed by B cells from the EGFR-negative group than EGFR-positive group (figure 6F). These data indicated that B cells from the EGFR-negative group might have been recruited by CHIT1_TAM and had the potential to construct the TLS in the TME, consistent with other findings from our study.

\section{DCs and other cells help promote an immunosuppressive TME in EGFR-mutant LUAD}

DCs also modify the TME and regulate the antitumor immune response. We distinguished $\mathrm{CD}_{1} \mathrm{~A}^{+} \mathrm{DC}, \mathrm{CD}_{1 \mathrm{C}}{ }^{+}$ DC, CLEC9A ${ }^{+}$DC, $\mathrm{LAMP}^{+}$DC, and pDC subclusters (online supplemental figure 7A,B). The proportions of all subclusters, but particularly $\mathrm{CD} 1 \mathrm{~A}^{+} \mathrm{DC}$ and $\mathrm{CD}_{\mathrm{C}}^{+}$ DC, were higher in the EGFR-positive group (online supplemental figure 7C). In mIHC, although the total proportion of $\mathrm{CD}_{1 \mathrm{C}}{ }^{+} \mathrm{DCs}$ was higher in the EGFR-positive group, the proportion in the stromal area was higher in the EGFR-negative group (online supplemental figure 7D).

Expression levels of CCL4, CCL17, CCL22, CXCL2, and CXCL17 were higher in DCs in the EGFR-positive group (online supplemental figure 7E). CCL17 and CCL22 are ligands of CCR4, and are related to the 


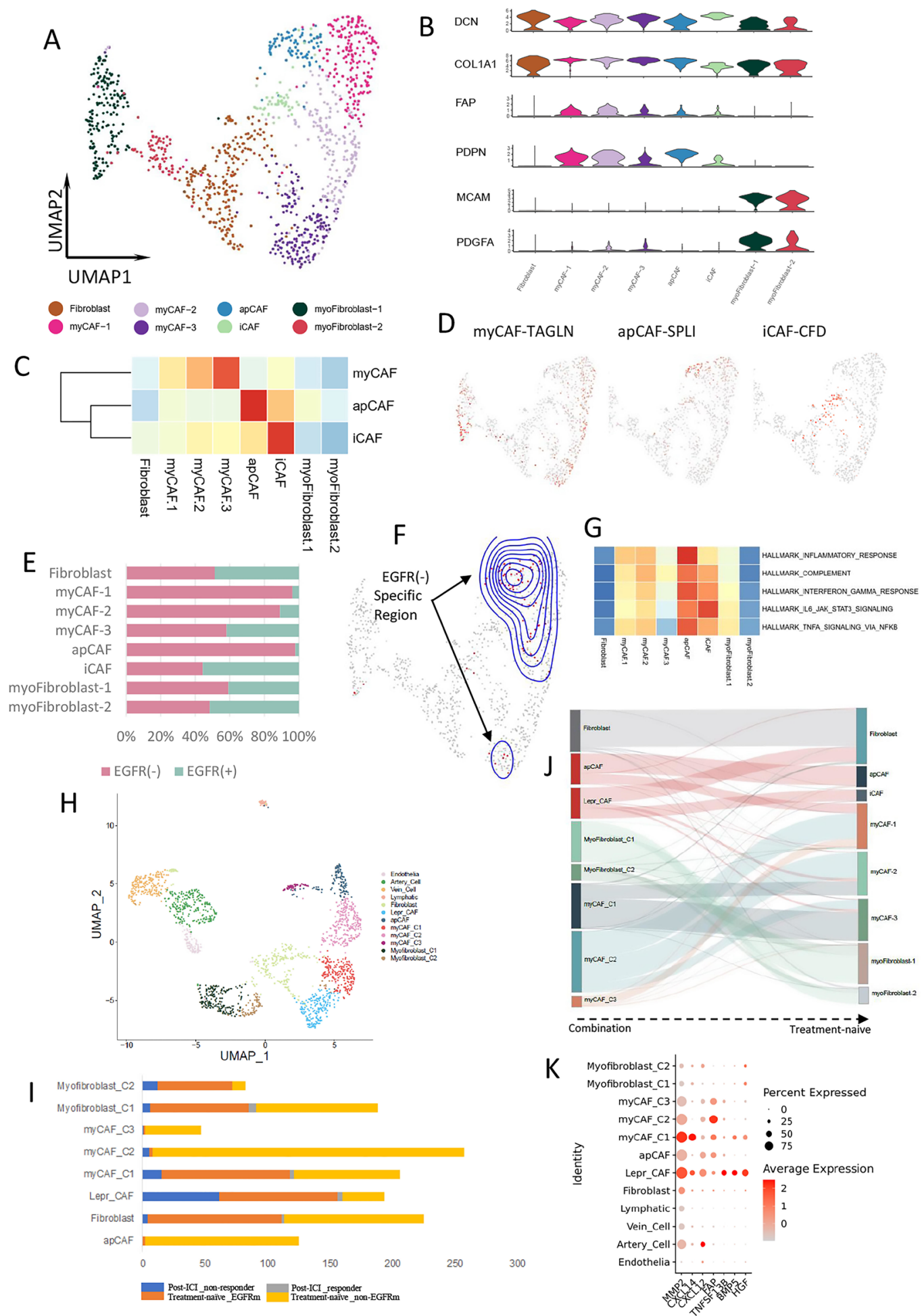

Figure 5 CAF clustering and function in EGFR-negative LUAD. (A) UMAP plot of 1145 fibroblasts from treatment-naïve samples. (B) Violin plot of different marker genes for each fibroblast cluster. (C) The correlation of each fibroblast cluster with different CAF subtypes according to the marker gene expression scoring. (D) Expression of marker genes for myCAFs, apCAFs, and iCAF. (E) The proportion of each fibroblast cluster in EGFR(-) (pink) and EGFR(+) (cyan) group. (F) UMAP plot of fibroblast from EGFR(-)-specific region calculated by DASeq. (G) Heatmap of the Qusage GO term enrichment/hallmark analysis for different fibroblast clusters. (H) UMAP plot of pooled fibroblast and endothelial cells in both treatment-naïve and post-treatment samples. (I) The proportion of different fibroblast cluster from post-ICI responder, post-ICI non-responder, treatment-naïve EGFR-mutant, and treatment-naïve EGFR-negative samples, respectively. $(\mathrm{J})$ Fibroblast clusters comparation between combination samples and treatment-naïve samples. (K) Bubble plot of the MSC-associated marker genes expressed by LEPR ${ }^{+}$ CAFs. apCAFs, antigen presenting CAFs; DASeq, differential abundance sequencing; EGFR, epidermal growth factor receptor; CAFs, cancer-associated fibroblasts; GO, Gene Ontology; iCAFs, inflammatory CAFs; ICI, immune-checkpoint inhibitor; LEPR leptin receptor, LUAD, lung adenocarcinoma; MSC, mesenchymal stem cell; myCAFs, myofibroblastic CAFs. 
A

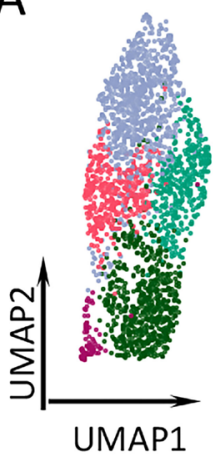

B MS4A1

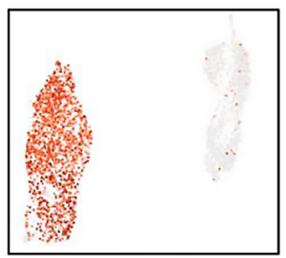

IGHG1
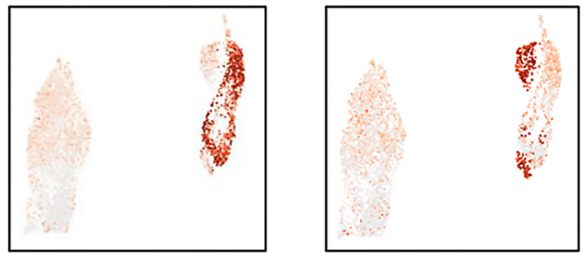

C

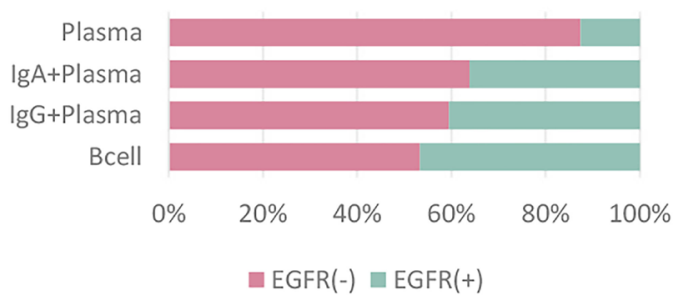

E

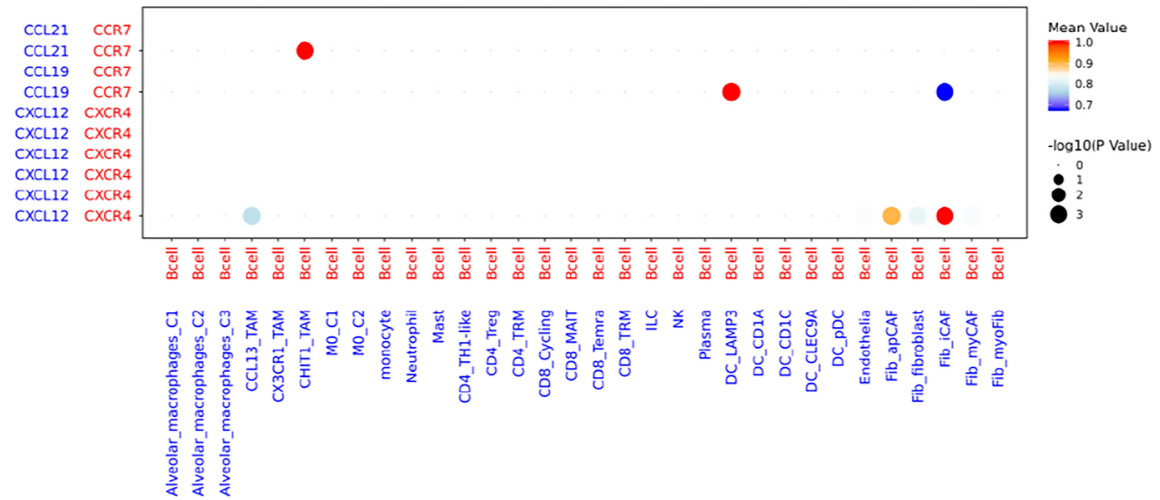

D

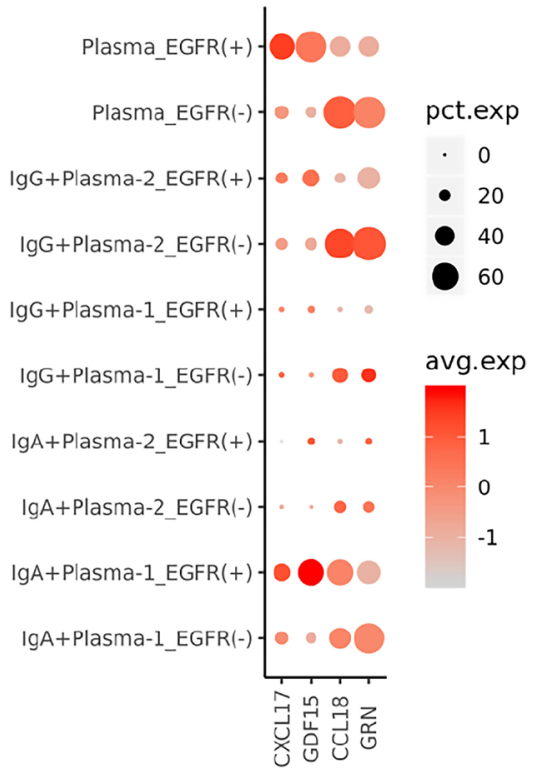

F

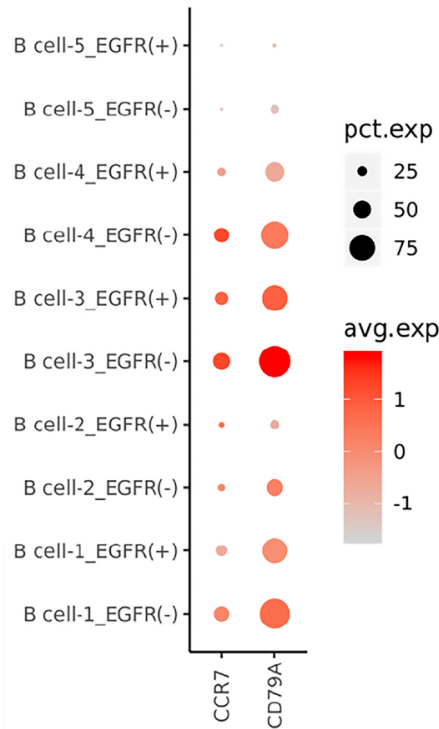

Figure 6 B-cell clustering and differential gene expression. (A) UMAP plot of 3453 B/plasma cells from treatment-naïve samples. (B) Expression of marker genes for different B/plasma cell clusters. (C) The proportion of each B/plasma cells cluster in EGFR(-) (pink) and EGFR(+) (cyan) group. (D) Bubble plot of differential gene expression among plasma cell clusters from different groups. (E) Cell-cell interactions between B cells and other types of immune cells through ligand and receptor binding. (F) Expression of CCR7 and CD79A among B cell clusters from different groups. EGFR, epidermal growth factor receptor.

increased population of Foxp $^{+}$Tregs in tumors. ${ }^{47}$ CCL4, CXCL2, and CXCL17 are chemoattractants for immune-suppressive cells, including MDSCs, Tregs, and macrophages. ${ }^{21-23}$

Neutrophils have seldom been reported in previous studies using $10 \times$ Genomics technology, due to its limited capability to capture neutrophils. ${ }^{48}$ We used BD technology to analyze neutrophils in the TME of LUAD. The proportion of neutrophils was lower in EGFR-mutant LUAD than in EGFR wild-type LUAD (figure 1B,E). Tumor-associated neutrophils increase T cell IFN- $\gamma$ production and activation, and promote their proliferation by increasing the 
expression of CD54 (ICAM1) and decreasing that of CD62L (SELL), CXCR1, and CXCR2. ${ }^{49}$ However, neutrophils in the EGFR-positive group exhibited downregulation of CD54 and upregulation of CD62L, CXCR1, and CXCR2. Moreover, the expression of CXCL8, a neutrophil chemoattractant, was lower in neutrophils in the EGFR-positive group (online supplemental figure 8A). Therefore, the functionality and infiltration of neutrophils were decreased in the TME of the EGFR-positive LUAD.

Endothelial cells were classified into eight subclusters and annotated as endothelial progenitor, artery, vein, capillary, and lymphatic cells (online supplemental figure $8 \mathrm{~B}, \mathrm{C})$. The proportion of endothelial cells was higher in the EGFR-positive group in all subclusters except for lymphatic cells (online supplemental figure 8D). Expression of CXCL12, CCL2, and CXCL17 by endothelial cells was higher in the EGFR-positive group, and that of CCL18 and CXCL2 was higher in the EGFR-negative group (online supplemental figure 8E). CCL2 and CXCL17 are immune-suppressive cytokines that attract MDSCs and macrophages, respectively. ${ }^{22} 24$

Mast cells were mainly detected in EGFR-positive samples (figure 1B,E). Mast cells contribute to angiogenesis, and induce neovascularization by releasing proangiogenic factors. ${ }^{50}$ The proangiogenic gene vascular endothelial growth factor-A (VEGFA) was highly expressed by mast cells in our samples, showing the important function of these cells (online supplemental figure $8 \mathrm{~F}$ ). Moreover, CSF1 was also found to be highly expressed by mast cells, indicating that mast cells might play an important role in regulating the migration, proliferation, and function of macrophages in the TME.

\section{Cellular interaction networks differed according to EGFR status}

We used CellPhoneDB to investigate cell-cell communication in the TME of LUAD. The cell communication type differed according to EGFR genetic status. As CD8 ${ }^{+}$ TRM cells might be the main immune cells responsible for the efficacy of immunotherapy, we analyzed the specific interactions between $\mathrm{CD} 8^{+}$TRM and other cell types (figure 7A). Interactions of $\mathrm{CD}^{+}{ }^{+} \mathrm{TRM}$ cells with CHIT1_TAM, LAMP3 ${ }^{+}$DCs and fibroblasts were noted in the EGFR-negative group. CHIT1_TAM can attract and activate TRM cells via CXCL9/CXCL10CXCR3 binding. ${ }^{28}{ }^{29} \mathrm{LAMP}^{+}$DC might facilitate TRM expansion and homeostatic survival through IL-15 and IL-15R binding in EGFR-negative groups. ${ }^{12}$ Meanwhile, fibroblasts in our EGFR-negative group also specifically interacted with TRM, via TGFB2-TGFBR2 and TGFB3TGFBR3 binding, which are essential for CD103 upregulation by $\mathrm{CD}^{+}{ }^{+} \mathrm{T}$ cells for conversion into TRM cells. ${ }^{44}$

We next used the CellPhoneDB to analyze the ligands, receptors, and their interactions between two specific cell subgroups with different EGFR status. We found some of the ligand-receptor binding pairs between two specific cell subgroups were sharing in both EGFR negative and positive groups, but some others were only appeared in either EGFR negative or positive group. Regarding the immune checkpoint ligand-receptor pairs, there were more specific interactions between $\mathrm{T}$ cells and other cell types, including PD-1 and PD-L1, in EGFR wild-type compared with EGFR-mutant LUAD (figure 7B-D). The IHC results showed lower expression of immune checkpoints, including PD-1, PD-L1, CTLA4, LAG3, TIM3, and CD47, in the EGFR-positive group (figure 7E). Of note, TIGIT was more highly expressed in the EGFR-positive group, especially in the stroma, with the opposite pattern seen for other immune checkpoints (figure 7E). These results may provide insight into possible immunotherapy strategies for EGFR-mutant LUAD.

\section{DISCUSSION}

The immune environment around EGFR-positive tumors of LUAD has been investigated previously. Using the Cancer Genome Atlas database, Zhong et al reported that patients with EGFR mutation lacked $\mathrm{T}$ cell infiltration and had a decreased proportion of PD-L $1^{+} / \mathrm{CD}^{+}$TILs. $^{5}$ EGFR-mutant NSCLC tumors reportedly have reduced expression levels of PD-1, LAG3, and TIM3 compared with EGFR wild-type tumors. ${ }^{51}$ Toki et $a l^{\beta}$ reported PD-L1 expression was significantly lower in EGFR mutant NSCLC patients compared with EGFR/KRAS WT, TILs presented in EGFR mutant tumors are more commonly inactive, and PD-L1 expression cannot reflect the underlying T cell activity in EGFR-driven NSCLC. All these conclusions could be partially supported by our findings in the current study. However, these studies are all explained the TME characteristics of EGFR mutant NSCLC from one or several aspects. To the best of our knowledge, no previous study has investigated the specific immune landscape of LUAD with EGFR mutation from single cell level.

In our study, we have identified some cell subtypes might be associated to better response of immune therapy, which specifically existed in the treatment-naïve EGFR wild-type samples. In order to validate whether these cell subtypes existed in the responsive samples after immunotherapy and had functional effect to immune response, we intended to analyze scRNA-seq data from samples after treatment of ICIs. Fortunately, we found that there were three samples after immune therapy sequenced by scRNA-seq and had clinical response after ICI treatment. After integrating the data of these three samples and ours, we could find some cell subtypes that existed in both the post-ICI responsive sample and EGFR-negative treatmentnaive samples, but not in post-ICI non-responsive samples and EGFR-positive treatment-naïve samples. Although the external dataset was only from three nodules in one patient, it was the only single cell sequencing dataset from LUAD samples post-PD-1 treatment with clinical response results which has been disclosed. Additionally, as the three samples with different responses to immunotherapy were coming from one patient, the extrinsic 

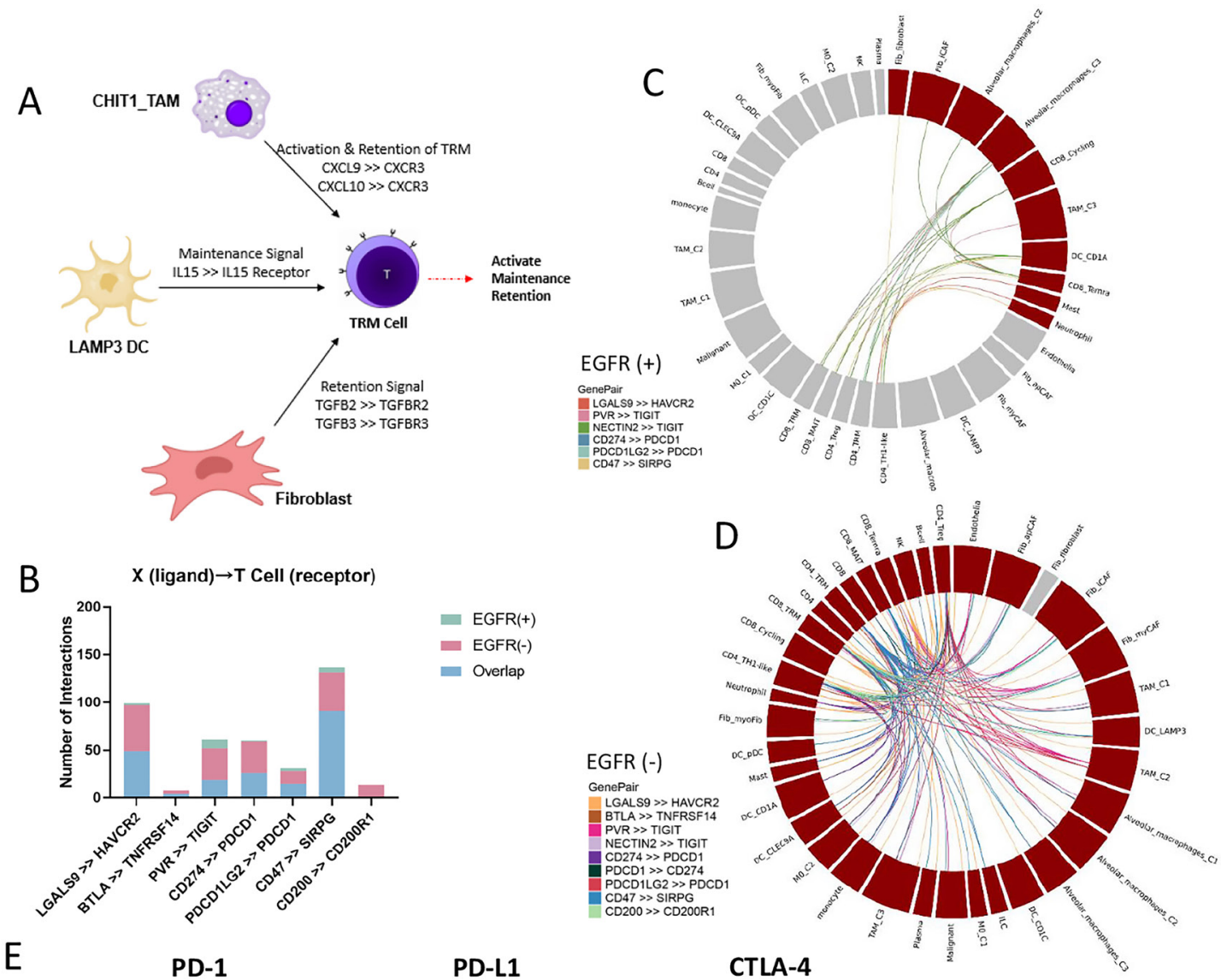

$E$

PD-1

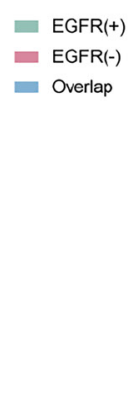

PD-L1

CTLA-4

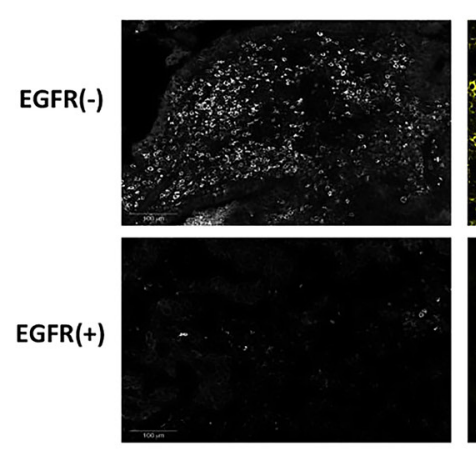

TIM-3

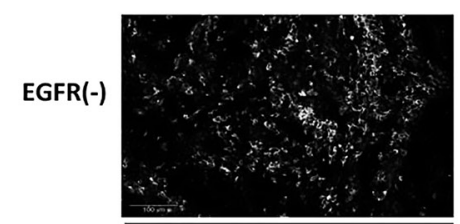

EGFR(+)
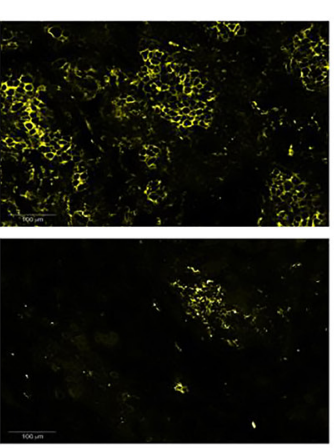

LAG-3
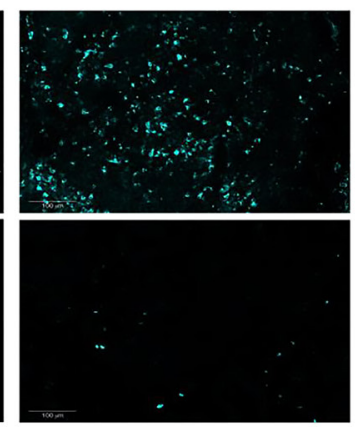
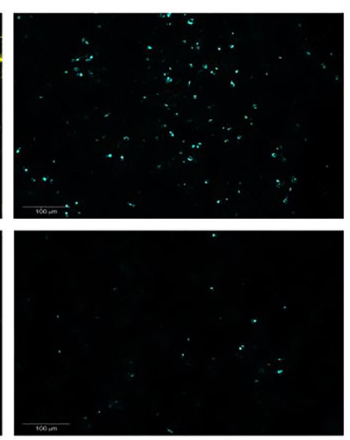

CD47

TIGIT
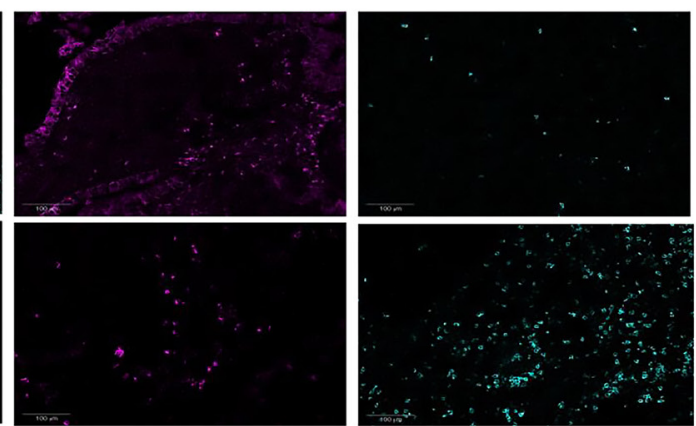

Figure 7 Cell interactions within the TME of LUAD. (A) The specific interactions between CD8 ${ }^{+}$TRM cells and other cells in EGFR(-) group. (B) Bar chart shows the interactions between T cells expressing immune checkpoint receptors and other cells that express immune checkpoint ligands in EGFR(+) and EGFR(-) group. (C) Circle plot of specific immune checkpoint interactions between T cell and other cells in EGFR(+) group. (D) Circle plot of specific immune checkpoint interactions between T cell and other cells in EGFR(-) group. (E) Immune checkpoint protein expression in EGFR(+) and EGFR(-) samples, including PD-1, PD-L1, CTLA4, TIM3, LAG3, CD47, and TIGIT. EGFR, epidermal growth factor receptor; LAG3, lymphocyte activating 3; LUAD, lung adenocarcinoma; PD-1, programmed cell death protein 1; PD-L1, programmed death-ligand 1; TIM3, T cell immunoglobulin and mucin domain-containing protein 3; TME, tumor microenvironment; TRM, tissue-resident memory. 
confounding factors could be ignored, which could help to reduce the bias.

The TME encompasses tumor and stromal cells, and is characterized by immune cells, endothelial cells, and fibroblasts and their interactions. Stromal cells are impacted by cancer cells and affect tumors by modulating angiogenesis, proliferation, invasion, and metastasis, as well as mediating therapeutic resistance. In this study, treatment-naïve malignant epithelial cells of EGFR wild-type LUAD showed similar characteristics to the epithelial cells of responders, and those of EGFR-mutant LUAD mapped onto the cells of non-responders. Thus, the EGFR gene status of tumor cells might play a decisive role in TME construction, by modifying communication among different cell types around the TME directly or indirectly.

TRM cell signature is associated with improved survival in lung cancer. ${ }^{17}$ Also, TRM is predictive of a response to immunotherapy in patients with triple-negative breast cancer or melanoma. ${ }^{16} 18$ In lung cancer, TRM cell was enriched in responders to PD-1 inhibitors, and in tumors with greater cytotoxic T cell responses. ${ }^{1752}$ TRM cells show high expression of genes encoding immunecheckpoint proteins, suggesting them to be a target of ICIs to restore the antitumor $\mathrm{T}$ cell response. The higher proportion of $\mathrm{CD}^{+} \mathrm{TRM}$ cells and higher expression of CXCL13 in treatment-naïve tumors of EGFR wild-type LUAD compared with EGFR-mutant LUAD supported the notion that EGFR mutation has a negative influence on the recruitment, activation, and retention of $\mathrm{CD} 8^{+}$ TRM cells, and on the generation of TLSs promoted by $\mathrm{CD}^{+}$TRM. This might involve communication between TRM and other cell types, including TAMs and CAFs.

TAMs can be classified into tumor-inhibiting M1 and tumor-promoting M2 phenotypes, and transition dynamically according to the environment. In $25 \%$ of patients with early stage lung cancer, M2-like TAMs also co-express M1-like macrophages. ${ }^{30}$ In this study, we annotated the TAMs based on their function rather than phenotype, and identified a subgroup of TAMs showing CXCL9 ${ }^{+}$/ CXCL10 ${ }^{+}$expression in EGFR wild-type LUAD; these may facilitate TRM recruitment and retention directly, and promote TRM expansion indirectly by secreting CCL7.

Moreover, CAFs in EGFR wild-type LUAD might facilitate the conversion of $\mathrm{CD}^{+} \mathrm{T}^{-} \mathrm{CD} 8^{+} \mathrm{TRM}$ cells in the TME by secreting TGF- $\beta$, while CAFs in EGFR-mutant LUAD might exhibit characteristics of MSCs. Finally, the core component of the TLS, B cells, had a greater tendency to be recruited by TAMs specifically from EGFR wild-type LUAD, and to generate TLS in the TME of EGFR wild-type LUAD.

The global single-cell landscape demonstrates the heterogeneity of cell components, function, and interaction between EGFR-mutant and wild-type LUADs. The lack of proinflammatory cells, enrichment of suppressive cell types, and lower expression of immune checkpoint proteins might cause EGFR-mutant LUAD having an immune-silent environment. Nonetheless,
EGFR-mutated NSCLC is a heterogenous subgroup of patients that responds differently to ICIs by allele. Previous study showed the exon 21 L858R groups were more inflamed tumors compared with exon 19 deletions, with higher $\mathrm{CD} 4{ }^{+}$and $\mathrm{CD} 8^{+}$expression. ${ }^{3}$ In our study, the small sample size limited performing the stratification by different sites of EGFR mutation even for the common mutations, and some analysis biases were inevitable.

Regarding the small sample size, our major findings have been tested by statistic method and supported by different cellular component crossover analysis such as the results related to CD8 ${ }^{+}$TRM. Moreover, we have integrated and compared the publicly released scRNA-seq data of postimmunotherapy samples to our own data of treatmentnaive samples, and we have also performed mIHC to confirm some of our most important results derived from scRNA-seq to strengthen the credibility of our findings. Thus, although our sample size was not large, and we did not analyze the dynamic characteristics of TME in paired samples before and after immunotherapy from single-cell perspective due to the limitation that patients with EGFR mutation are not recommended to receive ICI treatment, our study has still had some meaningful and interesting findings that might provide some hints for further studies and revealed some potential directions for future immunotherapy of EGFR-mutant LUAD.

\section{Conclusion}

EGFR-mutant LUAD has a specific TME quite different from that in EGFR wild-type LUAD. EGFR mutant tumor cells can recruit various immunosuppressive cells by secreting cytokines. The insufficiency of activated immune cells, including CD8 ${ }^{+}$TRM and CXCL9 ${ }^{+}$TAM, the high proportion of MSC-like CAFs, and the lower expression of immune checkpoint cooperatively tend to form a specific microenvironment in EGFR-mutant LUAD. In the future, combination immunotherapy can be considered for EGFR-mutant LUAD to improve the suppressive tumor immune microenvironment.

Acknowledgements We would like to thank the patients and their families for consenting tumor acquisition in our study. We thank Jiaying Zhou, Qiumei Deng and Jiayi Deng for tumor samples collection. We also thank Chao Zhang for data discussion.

Contributors $Q Z$ and $L Y$ designed the experiment. $L Y$ and $Y-T H$ analyzed and interpreted the data. SD and X-WW assisted the data and figure integration. Z-HC and X-RY collected the clinical samples and prepared single-cell suspension for sequencing. FW participated in the data analysis. BZ and W-DC performed bioinformatics analysis. X-MS performed multiplex immunohistochemistry examination and analysis. W-ZZ provided methodology, reviewing and editing. QZ and Y-LW conceived and supervised the study. LY wrote the manuscript with all authors' contribution to writing and providing feedbacks. QZ acted as guarantor and corresponding author for this study.

Funding The study was supported by the National Natural Science Foundation of China (grant number: 82072562 to QZ), the High-level Hospital Construction Project (grant number: DFJH201810 to QZ) and GDPH Scientific Research Funds for Leading Medical Talents in Guangdong Province (grant number: KJ012019428 to QZ).

Competing interests QZ reports honoraria from AstraZeneca, Boehringer Ingelheim, BMS, Eli Lilly, MSD, Pfizer, Roche, and Sanofi, outside the submitted work. WZ reports honoraria from AstraZeneca, Eli Lilly, Pfizer, Roche, and Sanofi, 
outside the submitted work. Y-LW reports advisory services for AstraZeneca, Boehringer Ingelheim, Novartis, and Takeda; personal fees from AstraZeneca, Beigene, Boehringer Ingelheim, BMS, Eli Lilly, MSD, Pfizer, Roche, and Sanofi; grants from AstraZeneca, Boehringer Ingelheim, BMS, Hengrui, and Roche, outside the submitted work. All other authors declare no competing interests.

\section{Patient consent for publication Not applicable.}

Ethics approval This study involves human participants and was approved by the Research Ethics Committee of Guangdong Provincial People's Hospital (reference number: GDREC2019304H(R1)). Participants gave informed consent to participate in the study before taking part; separate consent was obtained for tumor specimens for biomarker analyses.

\section{Provenance and peer review Not commissioned; externally peer reviewed.}

Data availability statement Data are available in a public, open access repository. Data are available upon reasonable request. Single-cell RNA sequencing datasets generated in this study are available on the GEO database under the accession number GSE171145. The public single-cell sequencing dataset applied in this study is also on the GEO database with number GSE146100. All other relevant data are available from the corresponding author of this study (QZ, gzzhouqing@126.com) upon reasonable request.

Supplemental material This content has been supplied by the author(s). It has not been vetted by BMJ Publishing Group Limited (BMJ) and may not have been peer-reviewed. Any opinions or recommendations discussed are solely those of the author(s) and are not endorsed by BMJ. BMJ disclaims all liability and responsibility arising from any reliance placed on the content. Where the content includes any translated material, BMJ does not warrant the accuracy and reliability of the translations (including but not limited to local regulations, clinical guidelines, terminology, drug names and drug dosages), and is not responsible for any error and/or omissions arising from translation and adaptation or otherwise.

Open access This is an open access article distributed in accordance with the Creative Commons Attribution Non Commercial (CC BY-NC 4.0) license, which permits others to distribute, remix, adapt, build upon this work non-commercially, and license their derivative works on different terms, provided the original work is properly cited, appropriate credit is given, any changes made indicated, and the use is non-commercial. See http://creativecommons.org/licenses/by-nc/4.0/.

\section{ORCID iDs}

Lei Yang http://orcid.org/0000-0001-6888-2036

Fen Wang http://orcid.org/0000-0002-4876-710X

Yi-Long Wu http://orcid.org/0000-0002-3611-0258

Qing Zhou http://orcid.org/0000-0002-0478-176X

\section{REFERENCES}

1 Topalian SL, Hodi FS, Brahmer JR, et al. Five-year survival and correlates among patients with advanced melanoma, renal cell carcinoma, or non-small cell lung cancer treated with nivolumab. JAMA Oncol 2019;5:1411-20.

2 Li J, Gu J. PD-L1 expression and EGFR status in advanced nonsmall-cell lung cancer patients receiving PD-1/PD-L1 inhibitors: a meta-analysis. Future Oncol 2019;15:1667-78.

3 Toki MI, Mani N, Smithy JW, et al. Immune marker profiling and programmed death ligand 1 expression across NSCLC mutations. $J$ Thorac Oncol 2018;13:1884-96.

4 Association between tissue TMB (tTMB) and clinical outcomes with pembrolizumab monotherapy (pembro) in PD-L1-positive advanced NSCLC in the KEYNOTE-010 and -042 trials. Ann Oncol 2019;30:v851-934.

5 Dong Z-Y, Zhang J-T, Liu S-Y, et al. EGFR mutation correlates with uninflamed phenotype and weak immunogenicity, causing impaired response to PD-1 blockade in non-small cell lung cancer. Oncoimmunology 2017;6:e1356145.

6 Reck M, Mok TSK, Nishio M, et al. Atezolizumab plus bevacizumab and chemotherapy in non-small-cell lung cancer (IMpower150): key subgroup analyses of patients with EGFR mutations or baseline liver metastases in a randomised, open-label phase 3 trial. Lancet Respir Med 2019;7:387-401.

7 Zhang C, Yin K, Liu S-Y, et al. Multiomics analysis reveals a distinct response mechanism in multiple primary lung adenocarcinoma after neoadjuvant immunotherapy. J Immunother Cancer 2021;9:e002312.

8 Su S, Liao J, Liu J, et al. Blocking the recruitment of naive CD4 ${ }^{+}$ $\mathrm{T}$ cells reverses immunosuppression in breast cancer. Cell Res $2017 ; 27: 461-82$
9 Liao W, Overman MJ, Boutin AT, et al. KRAS-IRF2 axis drives immune suppression and immune therapy resistance in colorectal cancer. Cancer Cell 2019;35:559-72.

10 Miyake M, Hori S, Morizawa Y, et al. CXCL1-mediated interaction of cancer cells with tumor-associated macrophages and cancerassociated fibroblasts promotes tumor progression in human bladder cancer. Neoplasia 2016;18:636-46

11 Apte RN, Voronov E. Immunotherapeutic approaches of IL-1 neutralization in the tumor microenvironment. J Leukoc Biol 2017;102:293-306

12 Raeber ME, Zurbuchen Y, Impellizzieri D, et al. The role of cytokines in T-cell memory in health and disease. Immunol Rev 2018;283:176-93.

13 Timperi E, Focaccetti C, Gallerano D, et al. IL-18 receptor marks functional CD8 ${ }^{+} \mathrm{T}$ cells in non-small cell lung cancer. Oncoimmunology 2017;6:e1328337.

14 Yang B, Wang X, Ren X. Amino acid metabolism related to immune tolerance by MDSCs. Int Rev Immunol 2012;31:177-83.

15 Cham CM, Driessens G, O'Keefe JP, et al. Glucose deprivation inhibits multiple key gene expression events and effector functions in CD8+ T cells. Eur J Immunol 2008;38:2438-50.

16 Edwards J, Wilmott JS, Madore J, et al. CD103 ${ }^{+}$tumor-resident CD8 $\mathrm{T}$ cells are associated with improved survival in immunotherapyNaïve melanoma patients and expand significantly during anti-PD-1 treatment. Clin Cancer Res 2018;24:3036-45.

17 Corgnac S, Malenica I, Mezquita L, et al. CD103 ${ }^{+} \mathrm{CD} 8^{+} \mathrm{T}_{\mathrm{RM}}$ cells accumulate in tumors of Anti-PD-1-responder lung cancer patients and are tumor-reactive lymphocytes enriched with Tc17. Cell Rep Med 2020;1:100127.

18 Byrne A, Savas P, Sant S, et al. Tissue-resident memory T cells in breast cancer control and immunotherapy responses. Nat Rev Clin Oncol 2020;17:341-8.

19 Workel HH, Lubbers JM, Arnold R, et al. A transcriptionally distinct $\mathrm{CXCL} 13^{+} \mathrm{CD}_{103}{ }^{+} \mathrm{CD} 8^{+} \mathrm{T}$-cell population is associated with $\mathrm{B}$-cell recruitment and neoantigen load in human cancer. Cancer Immunol Res 2019;7:784-96.

20 Helmink BA, Reddy SM, Gao J, et al. B cells and tertiary lymphoid structures promote immunotherapy response. Nature 2020;577:549555.

21 Zhang $\mathrm{H}$, Ye Y-L, Li M-X, et al. CXCL2/MIF-CXCR2 signaling promotes the recruitment of myeloid-derived suppressor cells and is correlated with prognosis in bladder cancer. Oncogene 2017;36:2095-104.

22 Burkhardt AM, Maravillas-Montero JL, Carnevale CD, et al. CXCL17 is a major chemotactic factor for lung macrophages. J Immunol 2014;193:1468-74.

23 Blattner C, Fleming V, Weber R, et al. CCR5 ${ }^{+}$myeloid-derived suppressor cells are enriched and activated in melanoma lesions. Cancer Res 2018;78:157-67.

24 Huang B, Lei Z, Zhao J, et al. CCL2/CCR2 pathway mediates recruitment of myeloid suppressor cells to cancers. Cancer Lett 2007;252:86-92.

25 Gjorgjevski M, Hannen R, Carl B, et al. Molecular profiling of the tumor microenvironment in glioblastoma patients: correlation of microglia/macrophage polarization state with metalloprotease expression profiles and survival. Biosci Rep 2019;39 doi:10.1042/ BSR20182361

26 Ratnam NM, Peterson JM, Talbert EE, et al. NF- $\kappa B$ regulates GDF-15 to suppress macrophage surveillance during early tumor development. J Clin Invest 2017;127:3796-809.

27 Han KY, Kim CW, Lee TH, et al. CCL23 up-regulates expression of KDR/Flk-1 and potentiates VEGF-induced proliferation and migration of human endothelial cells. Biochem Biophys Res Commun 2009;382:124-8.

28 Dangaj D, Bruand M, Grimm AJ, et al. Cooperation between constitutive and inducible chemokines enables T cell engraftment and immune attack in solid tumors. Cancer Cell 2019;35:885-900.

29 Andersson A, Srivastava MK, Harris-White M, et al. Role of CXCR3 ligands in IL-7/IL-7R alpha-Fc-mediated antitumor activity in lung cancer. Clin Cancer Res 2011;17:3660-72.

30 Garrido-Martin EM, Mellows TWP, Clarke J, et al. M1 ${ }^{\text {hot }}$ tumorassociated macrophages boost tissue-resident memory $\mathrm{T}$ cells infiltration and survival in human lung cancer. $J$ Immunother Cancer 2020;8.

31 House IG, Savas P, Lai J, et al. Macrophage-derived CXCL9 and CXCL10 are required for antitumor immune responses following immune checkpoint blockade. Clin Cancer Res 2020;26:487-504.

32 Tan C, Hiwa R, Mueller JL, et al. NR4A nuclear receptors restrain B cell responses to antigen when second signals are absent or limiting. Nat Immunol 2020;21:1267-79. 
33 Zhang M, Yang W, Wang P, et al. CCL7 recruits CDC1 to promote antitumor immunity and facilitate checkpoint immunotherapy to nonsmall cell lung cancer. Nat Commun 2020;11:6119.

34 Zhou BO, Yue R, Murphy MM, et al. Leptin-receptor-expressing mesenchymal stromal cells represent the main source of bone formed by adult bone marrow. Cell Stem Cell 2014;15:154-68.

35 Ye Y, Kuang X, Xie Z, et al. Small-molecule MMP2/MMP9 inhibitor SB-3CT modulates tumor immune surveillance by regulating PD-L1. Genome Med 2020;12:83.

36 Sjöberg E, Meyrath M, Milde L, et al. A novel ACKR2-dependent role of fibroblast-derived CXCL14 in epithelial-to-mesenchymal transition and metastasis of breast cancer. Clin Cancer Res 2019;25:3702-17.

37 Costa A, Kieffer Y, Scholer-Dahirel A, et al. Fibroblast heterogeneity and immunosuppressive environment in human breast cancer. Cancer Cell 2018;33:463-79.

38 Feig C, Jones JO, Kraman M, et al. Targeting CXCL12 from FAPexpressing carcinoma-associated fibroblasts synergizes with antiPD-L1 immunotherapy in pancreatic cancer. Proc Natl Acad Sci U S A 2013;110:20212-7.

39 Yarchoan M, Ho WJ, Mohan A, et al. Effects of B cell-activating factor on tumor immunity. JCl Insight 2020;5 doi:10.1172/jci. insight. 136417

40 Tremblay M, Viala S, Shafer ME, et al. Regulation of stem/progenitor cell maintenance by BMP5 in prostate homeostasis and cancer initiation. Elife 2020;9 doi:10.7554/eLife.54542

41 Papaccio F, Della Corte CM, Viscardi G, et al. Hgf/Met and the immune system: relevance for cancer immunotherapy. Int J Mol Sci 2018;19 doi:10.3390/ijms19113595

42 Maeda K, Enomoto A, Hara A, et al. Identification of meflin as a potential marker for mesenchymal stromal cells. Sci Rep 2016:6:22288.

43 Shangguan L, Ti X, Krause U, et al. Inhibition of TGF- $\beta /$ Smad signaling by BAMBI blocks differentiation of human mesenchymal stem cells to carcinoma-associated fibroblasts and abolishes their protumor effects. Stem Cells 2012;30:2810-9.

44 Mami-Chouaib F, Blanc C, Corgnac S, et al. Resident memory T cells, critical components in tumor immunology. $\mathrm{J}$ Immunother Cancer 2018;6:87.

45 Dong T, Yang D, Li R, et al. PGRN promotes migration and invasion of epithelial ovarian cancer cells through an epithelial mesenchymal transition program and the activation of cancer associated fibroblasts. Exp Mol Pathol 2016;100:17-25.

46 Gao J, Navai N, Alhalabi O, et al. Neoadjuvant PD-L1 plus CTLA-4 blockade in patients with cisplatin-ineligible operable high-risk urothelial carcinoma. Nat Med 2020;26:1845-51.

47 Mizukami Y, Kono K, Kawaguchi Y, et al. CCL17 and CCL22 chemokines within tumor microenvironment are related to accumulation of Foxp3+ regulatory T cells in gastric cancer. Int $J$ Cancer 2008;122:2286-93.

48 Zhu YP, Padgett L, Dinh HQ, et al. Identification of an early Unipotent neutrophil progenitor with pro-tumoral activity in mouse and human bone marrow. Cell Rep 2018;24:2329-41.

49 Eruslanov EB, Bhojnagarwala PS, Quatromoni JG, et al. Tumorassociated neutrophils stimulate $T$ cell responses in early-stage human lung cancer. J Clin Invest 2014;124:5466-80.

50 Komi DEA, Redegeld FA. Role of mast cells in shaping the tumor microenvironment. Clin Rev Allergy Immunol 2020;58:313-25.

51 Datar I, Sanmamed MF, Wang J, et al. Expression analysis and significance of PD-1, LAG-3, and Tim-3 in human non-small cell lung cancer using spatially resolved and multiparametric single-cell analysis. Clin Cancer Res 2019;25:4663-73.

52 Ganesan A-P, Clarke J, Wood O, et al. Tissue-resident memory features are linked to the magnitude of cytotoxic $T$ cell responses in human lung cancer. Nat Immunol 2017;18:940-50. 